\title{
ON THE DIFFUSIVE PROFILES FOR THE SYSTEM OF COMPRESSIBLE ADIABATIC FLOW THROUGH POROUS MEDIA*
}

\author{
PIERANGELO MARCATI ${ }^{\dagger}$ AND RONGHUA PAN $\ddagger$
}

\begin{abstract}
We study the Cauchy problem for the system of one dimensional compressible adiabatic flow through porous media and the related diffusive problem. We introduce a new approach which combines the usual energy methods with special $L^{1}$-estimates and use the weighted Sobolev norms to prove the global existence and large time behavior for the solutions of the problems. The asymptotic states for the solutions are given by either stationary solutions or similarity solutions depending on the behavior of the initial data when $|x| \rightarrow \infty$. Our estimates provide asymptotic time decay rates.
\end{abstract}

Key words. damping mechanism, diffusive profile, $L^{1}$-estimates, weighted energy estimates, decay rates

AMS subject classifications. 35L45, 76S05, 35Q35, 35K55

PII. S0036141099364401

1. Introduction. The motion of the adiabatic gas flow through porous media can be modeled by the following damped hyperbolic system:

$$
\left\{\begin{array}{l}
v_{t}-u_{x}=0, \\
u_{t}+p(v, s)_{x}=-\alpha u, \alpha>0, \\
\left(e(v, s)+\frac{1}{2} u^{2}\right)_{t}+(p u)_{x}=-\alpha u^{2} .
\end{array}\right.
$$

Where $v$ denotes the specific volume, $u$ is the velocity, $s$ stands for entropy, $p$ denotes the gas pressure with $p_{v}(v, s)<0$ for $v>0$, and $e$ is the specific internal energy for which one has $e_{s} \neq 0$ and $e_{v}+p=0$ (due to the second law of thermodynamics). For smooth solutions, the system (1.1) is equivalent to the following one:

$$
\left\{\begin{array}{l}
v_{t}-u_{x}=0, \\
u_{t}+p(v, s)_{x}=-\alpha u, \alpha>0, \\
s_{t}=0 .
\end{array}\right.
$$

It is strictly hyperbolic with characteristic speeds $-\lambda_{1}=\lambda_{3}=\sqrt{-p_{v}}$ and $\lambda_{2}=0$.

In this paper, we are interested in the influence of the damping mechanism to the smoothness and the large time behavior of the solutions. We study the Cauchy problem for the system (1.2) with the following initial data:

$$
(v, u, s)(x, 0)=\left(v_{0}(x), u_{0}(x), s_{0}(x)\right), \quad x \in R,
$$

satisfying the limit conditions

$$
\left(v_{0}, u_{0}, s_{0}\right)(x) \rightarrow\left(v_{ \pm}, u_{ \pm}, s_{ \pm}\right) \text {as } x \rightarrow \pm \infty
$$

${ }^{*}$ Received by the editors December 3, 1999; accepted for publication (in revised form) October 4, 2000; published electronically December 18, 2001.

http://www.siam.org/journals/sima/33-4/36440.html

${ }^{\dagger}$ Dipartmento di Matematica Pura ed Applicata, Universita degli Studi di L'Aquila, 67100, L'Aquila, Italy (marcati@univaq.it). The work of this author was supported in part by the European TMR project under contract ERBFMRX-CT 96-0033.

${ }^{\ddagger}$ SISSA, Via Beirut n. 2-4, 34014 Trieste, Italy. Present address, Department of Mathematics, University of Michigan, Ann Arbor, MI 48109-1109 (panrh@math.lsa.umich.edu). 
with $v_{ \pm}>0$. For the sake of simplicity, from now on, we take $\alpha=1$ and $p(v, s)=$ $(\gamma-1) v^{-\gamma} e^{s}$, with $\gamma>1$, which is the case for the polytropic gas dynamics.

The global existence with small initial data of smooth solutions for the Cauchy problem (1.2)-(1.3) has been studied first in [10] and [11] and later by [22]. Then a natural problem is the large time behavior of the solutions. From asymptotic analysis, it is known that the first term of $(1.2)_{2}$ decays to zero, as $t \rightarrow \infty$, faster than others. Therefore it is natural to expect that the problem (1.2)-(1.3) is time-asymptotically equivalent to the following reduced problem:

$$
\left\{\begin{array}{l}
\tilde{v}_{t}=-p(\tilde{v}, s)_{x x} \\
\tilde{u}=-p(\tilde{v}, s)_{x} \\
s_{t}=0 \\
\tilde{v}(x, 0)=\tilde{v}_{0}(x), s(x, 0)=s_{0}(x) \\
\tilde{v}_{0}( \pm \infty)=v_{ \pm}, s_{0}( \pm \infty)=s_{ \pm} .
\end{array}\right.
$$

The system in (1.4) is obtained from (1.2) by approximating the momentum equation in (1.2) with Darcy's law. Since the first equation of (1.4) is parabolic, the damping mechanism in (1.2) creates some diffusive effects when $t$ tends to infinity.

For the isentropic flow, namely, $s=$ const, (1.2) takes the following form:

$$
\left\{\begin{array}{l}
v_{t}-u_{x}=0 \\
u_{t}+p(v)_{x}=-u
\end{array}\right.
$$

The diffusive effect created by the damping mechanism has been investigated for the Cauchy problem of (1.5) with the initial data

$$
(v(x, 0), u(x, 0))=\left(v_{*}(x), u_{*}(x)\right)
$$

such that

$$
\lim _{x \rightarrow \pm \infty}\left(v_{*}(x), u_{*}(x)\right)=\left(v_{ \pm}, u_{ \pm}\right) .
$$

It has been proved in [5] that the smooth solution of (1.5)-(1.6) can be described time-asymptotically by the solution of the following parabolic problem:

$$
\left\{\begin{array}{l}
\tilde{v}_{t}=-p(\tilde{v})_{x x} \\
\tilde{u}=-p(\tilde{v})_{x} \\
\tilde{v}(x, 0)=\tilde{v}_{*}\left(x+d_{0}\right) .
\end{array}\right.
$$

Where $\tilde{v}_{*}$ is the similarity solution of $(1.7)_{1}$ with $\tilde{v}_{*}( \pm \infty)=v_{ \pm}$. For other results, we refer to [3], [4], [6], and [19] for smooth solutions and to [1], [4], [8], [12], [13], [14], [15], [17], [18], and [21] for weak solutions. For the initial boundary value problems on a quarter plane, we refer to [16] and [20].

There are few results in the literature for the case $s \neq$ const. Partial answers are given in [11] and [7] for the Cauchy problem and in the recent paper of Hsiao and Pan [9] concerning the initial boundary value problem. The case $v_{-}=v_{+}=\bar{v}$ and $s_{-}=s_{+}=\bar{s}$ was investigated in [11] and the case $v_{-} \neq v_{+}$and $s_{-}=s_{+}=\bar{s}$ was treated in [7] by using a technical condition (that they refer to in [7] as condition V) which requires us to solve the following parabolic problem

$$
\left\{\begin{array}{l}
\tilde{v}_{t}=-p(\tilde{v}, s)_{x x}, \\
\tilde{v}_{0}(x)=e^{\frac{1}{\gamma}(s(x)-\bar{s})} \tilde{v}_{*}\left(x+x_{0}\right)
\end{array}\right.
$$


and to control the behavior of its solutions by means of the similarity solutions of

$$
\left\{\begin{array}{l}
\tilde{v}_{* t}=-p\left(\tilde{v}_{*}, \bar{s}\right)_{x x} \\
\tilde{v}_{*}( \pm \infty)=v_{ \pm}
\end{array}\right.
$$

The purpose of this paper is to deal with the following two cases:

Case 1: $s_{-}=s_{+}=\bar{s}$;

Case 2: $\left(v_{ \pm}, s_{ \pm}\right)$satisfy $p\left(v_{-}, s_{-}\right)=p\left(v_{+}, s_{+}\right)=\bar{p}$.

In Case 1, namely, $s_{-}=s_{+}=\bar{s}$, we cannot use the methods of [7] and we need new techniques. In particular we shall combine the usual energy methods with special $L^{1}$ estimates and with the use of weighted Sobolev norms to solve the problems in detail. This is the only case where $p\left(v_{-}, s_{-}\right) \neq p\left(v_{+}, s_{+}\right)$that we can treat in this paper. In this case, the asymptotic states will be the similarity solution of (1.9) given by the scaling invariance with respect to the transformation $x \rightarrow \sigma x, t \rightarrow \sigma^{2} t$. Our results strongly improved those in [7]. Indeed, we remove the technical condition $\mathrm{V}$ and we describe the asymptotic states both for the diffusion problem and the hyperbolic one by using the similarity solutions. Thanks to our new approach, it is possible to get a decay rate which did not exist in the previous results (see [7]). Our results on the parabolic problem generalize the result of [2] to the adiabatic case, and also obtain better decay rates.

In Case 2, we can determine a special solution $v_{3}(x)$ to $(1.4)_{1}$ by solving the equation $p\left(v_{3}, s\right)=\bar{p}$. Then in this case we establish results similar to those obtained in [11] with, in addition, some decay rates.

Before stating the main results, we describe the plan of this paper. In section 2 , the parabolic problem (1.4) is studied in detail for both cases by using our new approach. Then sections 3 and 4 are devoted to the hyperbolic problem (1.2)-(1.3) for Case 1 and Case 2, respectively.

We now state our main results.

1.1. Main results: Parabolic equation. Since in (1.2) or (1.4) $s_{t}=0$, then $s(x, t)=s(x)=s_{0}(x)$. Let us denote

$$
\begin{aligned}
& a(x)=(\gamma-1)^{-\frac{1}{\gamma}} e^{-\frac{1}{\gamma} s(x)}, \\
& a_{1}=(\gamma-1)^{-\frac{1}{\gamma}} e^{-\frac{1}{\gamma} \bar{s}}, \\
& w \equiv a(x) \tilde{v}=p(\tilde{v}, s)^{-\frac{1}{\gamma}},
\end{aligned}
$$

then (1.4) is equivalent to the following:

$$
\left\{\begin{array}{l}
w_{t}+a(x)\left(w^{-\gamma}\right)_{x x}=0 \\
\tilde{u}=-\left(w^{-\gamma}\right)_{x} \\
s(x, t)=s_{0}(x) \\
w(x, 0)=w_{0}(x)=a(x) \tilde{v}_{0}(x) \\
w( \pm \infty)=w_{ \pm}>0
\end{array}\right.
$$

Moreover, we will denote by $\tilde{w}(\eta)$ (with $\eta=\frac{x}{\sqrt{t+1}}$ ) the similarity solution of the following problem:

$$
\left\{\begin{array}{l}
\tilde{w}_{t}+a_{1}\left(\tilde{w}^{-\gamma}\right)_{x x}=0 \\
\tilde{w}( \pm \infty)=w_{ \pm}
\end{array}\right.
$$


By combining the weighted energy method and $L^{1}$-estimate, we can prove the following theorem for Case 1.

Theorem 1.1. Assume that $w_{0}(x)$ and $s_{0}(x)$ are $C^{2}$ functions and

$$
w_{0}(x)-\tilde{w}(x, 0) \in H^{2}(R) \cap L^{1}(R), \quad x\left(s_{0}(x)-\bar{s}\right) \in L^{1}(R) .
$$

There exists $\delta_{0}>0$ such that, if $0<\delta<\delta_{0}$ and

$$
\left|w_{+}-w_{-}\right|+\left\|w_{0}(x)-\tilde{w}(x, 0)\right\|_{H^{2}} \leq \delta
$$

then (1.11) has a unique global smooth solution $(w, \tilde{u}, s)(x, t)$ satisfying

$$
w(x, t)-\tilde{w} \in C^{0}\left([0, t] ; H^{2}\right) \text { for all } t>0 .
$$

Moreover, there exist positive constants $C>0, \beta_{1}>\frac{1}{3}$, and $\beta_{2}>\frac{1}{2}$ such that

$$
\begin{aligned}
& \|w(x, t)-\tilde{w}\|_{L^{\infty}} \leq C(1+t)^{-\frac{1}{2}}(1+\log (1+t))^{\beta_{1}}, \\
& \left\|\tilde{u}+\left(\tilde{w}^{-\gamma}\right)_{x}\right\|_{L^{\infty}} \leq C(1+t)^{-1}(1+\log (1+t))^{\beta_{2}} .
\end{aligned}
$$

Thus, by setting $\tilde{v}=a^{-1} w, \hat{v}=a^{-1} \tilde{w}$, and $\hat{u}=-\left(\tilde{w}^{-\gamma}\right)_{x}$, one obtains the (unique) global smooth solution $(\tilde{v}, \tilde{u}, s)$ to (1.4) which satisfies

$$
\begin{aligned}
& \|\tilde{v}-\hat{v}\|_{L^{\infty}} \leq C(1+t)^{-\frac{1}{2}}(1+\log (1+t))^{\beta_{1}}, \\
& \|\tilde{u}-\hat{u}\|_{L^{\infty}} \leq C(1+t)^{-1}(1+\log (1+t))^{\beta_{2}} .
\end{aligned}
$$

Remark 1. (a) Our results in Theorem 1.1 generalize the ones in [2] to the adiabatic case and extend to a larger class of initial data. The decay rate here is better than in [2] and is almost optimal.

(b) The condition $x(s(x)-\bar{s}) \in L^{1}(R)$ can be replaced by the weaker one:

$$
|x|^{\beta}(s(x)-\bar{s}) \in L^{1}(R)
$$

for $\beta>0$. This is clear from our proof below.

For Case 2 , where $w_{-}=w_{+}=\bar{w}$ in $(1.11)$, it is clear that $\left(\bar{w}, 0, s_{0}(x)\right)$ is a special solution for the system in (1.11). Let us denote $v_{1}(x)=a^{-1} \bar{w}$ and we have the following.

Theorem 1.2. Assume that $w_{0}(x)$ and $s_{0}(x)$ are $C^{2}$ functions and $w_{0}-\bar{w} \in H^{2}$. There exists $\delta_{0}>0$ such that if $0<\delta<\delta_{0}$ and $\left\|w_{0}-\bar{w}\right\|_{H^{2}} \leq \delta$, then (1.11) has a unique global smooth solution $(w, \tilde{u}, s)(x, t)$ satisfying

$$
\lim _{t \rightarrow \infty}\|w(x, t)-\bar{w}\|_{L^{\infty}}=0 .
$$

Furthermore, if $w_{0}(x)-\bar{w} \in L^{1}$, then

$$
\begin{aligned}
& \|w(x, t)-\bar{w}\|_{L^{\infty}} \leq C(1+t)^{-\frac{1}{2}}(1+\log (1+t))^{\beta_{1}}, \\
& \|\tilde{u}\|_{L^{\infty}} \leq C(1+t)^{-1}(1+\log (1+t))^{\beta_{2}} .
\end{aligned}
$$

Thus, by setting $v_{2}=a^{-1} w$ and $u_{2}=\tilde{u}$, one has a unique global smooth solution $\left(v_{2}, u_{2}, s\right)$ to (1.4) such that

$$
\begin{aligned}
& \left\|v_{2}-v_{1}\right\|_{L^{\infty}} \leq C(1+t)^{-\frac{1}{2}}(1+\log (1+t))^{\beta_{1}} \\
& \left\|u_{2}\right\|_{L^{\infty}} \leq C(1+t)^{-1}(1+\log (1+t))^{\beta_{2}}
\end{aligned}
$$


1.2. Main results: Hyperbolic problems. Based on the results in the previous theorems, we can solve (1.2)-(1.3) in detail for both cases, respectively.

Following [5], we define

$$
\begin{aligned}
& m(x, t) \equiv-\left(u_{+}-u_{-}\right) m_{0}(x) e^{-t} \\
& u_{m}(x, t) \equiv u_{-} e^{-t}+\int_{-\infty}^{x} m_{t}(\xi, t) d \xi
\end{aligned}
$$

where $m_{0}(x)$ is a smooth function with compact support such that

$$
\int_{-\infty}^{+\infty} m_{0}(x) d x=1
$$

We first treat Case 1 , where $s_{-}=s_{+}=\bar{s}$. Denote by $(\tilde{v}, \tilde{u}, s)$ the solution to (1.4) obtained in Theorem 1.1. In addition, we assume

$$
\int_{-\infty}^{+\infty}\left(v_{0}(x)-\tilde{v}_{0}(x)\right) d x=-\left(u_{+}-u_{-}\right) .
$$

A special choice of $\tilde{v}_{0}$ is given in Remark 2 below. Let us denote $y(x, t)=\int_{-\infty}^{x}(v-$ $\tilde{v}-m)(\xi, t) d \xi$, then $y$ satisfies

$$
\left\{\begin{array}{l}
y_{t t}+\left[p\left(y_{x}+\tilde{v}+m, s\right)-p(\tilde{v}, s)\right]_{x}+y_{t}=p(\tilde{v}, s)_{x t} \\
y(x, 0)=y_{0}(x)=\int_{-\infty}^{x}\left(v_{0}(\xi)-\tilde{v}_{0}(\xi)-m(\xi, 0)\right) d \xi \\
y_{t}(x, 0)=y_{1}(x)=u_{0}(x)-\tilde{u}(x, 0)-u_{m}(x, 0) .
\end{array}\right.
$$

THEOREM 1.3. Under the conditions of Theorem 1.1, there exists $\varepsilon_{0}>0$ such that for all $0<\varepsilon<\varepsilon_{0}$ and $\left\|y_{0}\right\|_{H^{3}}+\left\|y_{1}\right\|_{H^{2}} \leq \varepsilon$, the system (1.15) admits a unique global smooth solution $y$ such that

$$
y \in C^{0}\left([0, t] ; H^{3}\right), \quad y_{t} \in C^{0}\left([0, t] ; H^{2}\right)
$$

for all $t>0$. Moreover, there exists $C=C(\varepsilon)>0$ such that

$$
\left\|y_{x}\right\|_{L^{\infty}} \leq C(1+t)^{-\frac{3}{4}},\left\|y_{t}\right\|_{L^{\infty}} \leq C(1+t)^{-\frac{5}{4}} .
$$

Hence, by setting $v(x, t)=\tilde{v}+m+y_{x}$ and $u(x, t)=\tilde{u}+u_{m}+y_{t}$, one has the (unique) global smooth solution $(v, u, s)$ to (1.2)-(1.3), such that

$$
\|v-\tilde{v}\|_{L^{\infty}} \leq C(1+t)^{-\frac{3}{4}},\|u-\tilde{u}\|_{L^{\infty}} \leq C(1+t)^{-\frac{5}{4}} .
$$

Furthermore, in view of Theorem 1.1, one has

$$
\begin{aligned}
& \|v-\hat{v}\|_{L^{\infty}} \leq C(1+t)^{-\frac{1}{2}}(1+\log (1+t))^{\beta_{1}}, \\
& \|u-\hat{u}\|_{L^{\infty}} \leq C(1+t)^{-1}(1+\log (1+t))^{\beta_{2}},
\end{aligned}
$$

where $\beta_{1}$ and $\beta_{2}$ are the same as before.

Remark 2. (a) The global existence for the smooth solution to (1.2)-(1.3) has been proved in [22], via characteristic method, provided that the initial data are small. We present here an alternative version in $H^{2}$ spaces by the energy estimate method. 
(b) (1.14) is the restriction on the initial data which comes from (1.2)-(1.3) and (1.4). This is also the case for (1.16) and (1.18) below. There is a large class of functions $\tilde{v}_{0}(x)$ which can be chosen (for any given $v_{0}(x)$ in (1.3)). A special choice is $\tilde{v}_{0}(x)=a^{-1} \tilde{w}\left(x+x_{0}, 0\right)$, where $x_{0}$ is uniquely determined by

$$
\int_{-\infty}^{+\infty}\left(v_{0}(x)-a^{-1} \tilde{w}\left(x+x_{0}, 0\right)\right) d x=-\left(u_{+}-u_{-}\right)
$$

which is the special case discussed in [7]. Since Theorem 1.1 is obviously valid for $w_{0}(x)=\tilde{w}\left(x+x_{0}, 0\right)$, we include the results of [7].

(c) A similar condition was used in [9] for the initial boundary problem related to (1.2), where it was proved that both the solutions to the damped hyperbolic problem and those of the related diffusive problem have the same time asymptotic states if the initial total excessive mass is zero.

Let us now consider Case 2. Assume that

$$
\int_{-\infty}^{+\infty}\left(v_{0}(x)-v_{2}(x, 0)\right) d x=-\left(u_{+}-u_{-}\right)
$$

and denote by $\tilde{y}(x, t)=\int_{-\infty}^{x}\left(v-v_{2}-m\right)(\xi, t) d \xi$, then we have

$$
\left\{\begin{array}{l}
\tilde{y}_{t t}+\left[\left(p\left(\tilde{y}_{x}+v_{2}+m, s\right)-p\left(v_{2}, s\right)\right]_{x}=p\left(v_{2}, s\right)_{x t},\right. \\
\tilde{y}(x, 0)=\tilde{y}_{0}(x)=\int_{-\infty}^{x}\left(v_{0}(\xi)-v_{2}(\xi, 0)-m(\xi, 0)\right) d \xi \\
\tilde{y}_{t}(x, 0)=\tilde{y}_{1}(x)=u_{0}(x)-u_{2}(x, 0)-u_{m}(x, 0) .
\end{array}\right.
$$

Similarly to Theorem 1.3, we have the following.

THEOREM 1.4. Under the hypotheses of the Theorem 1.2, there exists $\varepsilon_{0}>0$ such that if $0<\varepsilon<\varepsilon_{0}$ and $\left\|\tilde{y}_{0}\right\|_{H^{3}}+\left\|\tilde{y}_{1}\right\|_{H^{2}} \leq \varepsilon$, then (1.17) has a unique global smooth solution $\tilde{y}$ such that

$$
\tilde{y} \in C^{0}\left([0, t] ; H^{3}\right), \tilde{y}_{t} \in C^{0}\left([0, t] ; H^{2}\right)
$$

for all $t>0$. Moreover, there exists $C=C(\varepsilon)>0$ such that

$$
\left\|\tilde{y}_{x}\right\|_{L^{\infty}} \leq C(1+t)^{-\frac{3}{4}},\left\|\tilde{y}_{t}\right\|_{L^{\infty}} \leq C(1+t)^{-\frac{5}{4}} .
$$

Hence, by setting $v(x, t)=v_{2}+m+\tilde{y}_{x}$ and $u(x, t)=u_{2}+u_{m}+\tilde{y}_{t}$, one has the (unique) global smooth solution $(v, u, s)$ to (1.2)-(1.3); moreover,

$$
\left\|v-v_{2}\right\|_{L^{\infty}} \leq C(1+t)^{-\frac{3}{4}},\left\|u-u_{2}\right\|_{L^{\infty}} \leq C(1+t)^{-\frac{5}{4}} .
$$

Furthermore, in view of Theorem 1.2, one has

$$
\begin{aligned}
& \left\|v-v_{1}\right\|_{L^{\infty}} \leq C(1+t)^{-\frac{1}{2}}(1+\log (1+t))^{\beta_{1}}, \\
& \|u\|_{L^{\infty}} \leq C(1+t)^{-1}(1+\log (1+t))^{\beta_{2}} .
\end{aligned}
$$

Since $\left(v_{1}(x), 0, s(x)\right)$ is a special solution to both (1.2) and (1.4), if we assume

$$
\int_{-\infty}^{+\infty}\left(v_{0}-v_{1}\right)(x) d x=-\left(u_{+}-u_{-}\right)
$$


and denote by $z(x, t)=\int_{0}^{x}\left(v-v_{1}-m\right)(\xi, t) d \xi$, we have

$$
\left\{\begin{array}{l}
z_{t t}+\left[p\left(z_{x}+v_{1}+m, s\right)-p\left(v_{1}, s\right)\right]_{x}+z_{t}=0, \\
z(x, 0)=z_{0}(x)=\int_{-\infty}^{x}\left(v_{0}(\xi)-v_{1}(\xi)-m(\xi, 0)\right) d \xi \\
z_{t}(x, 0)=z_{1}(x)=u_{0}(x)-u_{m} .
\end{array}\right.
$$

Then in this special case, we have the following result, which includes the paper [11].

THEOREM 1.5. There exists $\varepsilon_{0}>0$ such that if $0<\varepsilon<\varepsilon_{0}$ and $\left\|z_{0}\right\|_{H^{3}}+\left\|z_{1}\right\|_{H^{2}} \leq$ $\varepsilon$, then (1.19) has a unique global smooth solution $z$ such that

$$
z \in C^{0}\left([0, t] ; H^{3}\right), z_{t} \in C^{0}\left([0, t] ; H^{2}\right)
$$

for all $t>0$. Moreover,

$$
\left\|z_{x}\right\|_{L^{\infty}} \leq C(1+t)^{-\frac{3}{4}},\left\|z_{t}\right\|_{L^{\infty}} \leq C(1+t)^{-\frac{5}{4}} .
$$

Hence, by setting $v(x, t)=v_{1}+m+z_{x}$ and $u(x, t)=u_{m}+z_{t}$, one has the (unique) global smooth solution $(v, u, s)$ to (1.2)-(1.3) such that

$$
\left\|v-v_{1}\right\|_{L^{\infty}} \leq C(1+t)^{-\frac{3}{4}},\|u\|_{L^{\infty}} \leq C(1+t)^{-\frac{5}{4}} .
$$

We will end this introduction by making a reduction. In fact, in sections 3 and 4 , we will only prove Theorems $1.3-1.5$ for the case $u_{-}=u_{+}=0$, where $m(x, t)=0$ and $u_{m}=0$. The general case can be treated in the similar way since $m(x, t)$ and $u_{m}$ decay to zero exponentially fast.

2. Nonlinear diffusion equation. This section is devoted to studing the diffusive problem (1.4). Clearly one has $s(x, t)=s_{0}(x) \equiv s(x)$ for all $t>0$, which then is sufficient to solve the following equation:

$$
\left\{\begin{array}{l}
\tilde{v}_{t}=-p(\tilde{v}, s)_{x x} \\
\tilde{v}(x, 0)=\tilde{v}_{0}(x), \tilde{v}_{0}( \pm \infty)=v_{ \pm}>0 .
\end{array}\right.
$$

The equation (2.1) is equivalent to the following porous media type equation:

$$
\left\{\begin{array}{l}
w_{t}+a(x)\left(w^{-\gamma}\right)_{x x}=0, \\
w(x, 0)=w_{0}(x)=a(x) \tilde{v}_{0}(x), \\
w( \pm \infty)=w_{ \pm}>0
\end{array}\right.
$$

where $a(x)=(\gamma-1)^{-\frac{1}{\gamma}} e^{-\frac{1}{\gamma} s(x)}, w \equiv a(x) \tilde{v}=p(\tilde{v}, s)^{-\frac{1}{\gamma}}$. We will study the equation (2.2) instead of (2.1) for the following two cases, which are equivalent to those stated in the introduction.

Case 1: $s_{-}=s_{+}=\bar{s}$.

Case 2: $\left(v_{ \pm}, s_{ \pm}\right)$are chosen such that $w_{-}=w_{+}=\bar{w}$, where we set $w_{ \pm}=$ $w\left(v_{ \pm}, s_{ \pm}\right)$.

We will concentrate our main efforts on Case 1 which is the most difficult part. 
2.1. Case 1: $s_{-}=s_{+}=\bar{s}$. In this subsection, (2.2) will be solved near the similarity solution for the related isentropic problem.

Now let us recall some results on the similarity solution for $(2.2)_{1}$ with $s(x)=$ const $=\bar{s}$. In this case, $(2.2)_{1}$ takes the form

$$
w_{t}+a_{1}\left(w^{-\gamma}\right)_{x x}=0
$$

with $a_{1}=(\gamma-1)^{-\frac{1}{\gamma}} e^{-\frac{1}{\gamma} \bar{s}}$. It is well known that (2.3) has a unique (up to a shift) similarity solution $\tilde{w}(\eta)$ (where $\eta=\frac{x}{\sqrt{1+t}}$ ) satisfying the limiting conditions $\tilde{w}( \pm \infty)=$ $w_{ \pm}$. Some properties of $\tilde{w}(\eta)$ are listed in the following lemma (see, for instance, [5]).

Lemma 2.1. Let $\tilde{w}(\eta)$ be the similarity solution to $(2.3)$ with $\tilde{w}( \pm \infty)=w_{ \pm}$and $\eta=\frac{x}{\sqrt{1+t}}$. It follows that

$$
\begin{gathered}
\left|\tilde{w}^{\prime}(\eta)\right|+\left|\tilde{w}^{\prime \prime}(\eta)\right| \leq C_{1}\left|w_{+}-w_{-}\right| \exp \left\{-C_{2} \eta^{2}\right\}, \\
\left|\tilde{w}(\eta)-w_{-}\right|_{\eta<0}+\left|\tilde{w}(\eta)-w_{+}\right|_{\eta>0} \leq C_{1}\left|w_{+}-w_{-}\right| \exp \left\{-C_{2} \eta^{2}\right\}, \\
\tilde{w}_{x}=(1+t)^{-\frac{1}{2}} \tilde{w}^{\prime}(\eta), \tilde{w}_{t}=-\frac{1}{2}(1+t)^{-1} \eta \tilde{w}^{\prime}(\eta),\left(\tilde{w}^{-\gamma}\right)_{x x}=-a_{1}^{-1} \tilde{w}_{t}, \\
\left\|D_{t}^{i} D_{x}^{j} \tilde{w}(\cdot, t)\right\|^{2} \leq C\left|w_{+}-w_{-}\right|^{2}(1+t)^{-(2 i+j)+\frac{1}{2}}, \\
\left\|D_{t}^{i} D_{x}^{j} \tilde{w}(\cdot, t)\right\|_{L^{\infty}} \leq C_{1}\left|w_{+}-w_{-}\right|(1+t)^{-\left(i+\frac{1}{2} j\right)}
\end{gathered}
$$

for $i+j \geq 1$ and $i \geq 0, j \geq 0$.

We now prove Theorem 1.1 by comparing $w(x, t)$ with $\tilde{w}(\eta)$.

Let us denote $\phi=w-\tilde{w}$; then from (2.2) and (2.3) we have the following equation:

$$
\left\{\begin{array}{l}
\phi_{t}+a(x)(\psi(\tilde{w}) \phi)_{x x}+\left(a-a_{1}\right)\left(\tilde{w}^{-\gamma}\right)_{x x}+a(x)\left(g(\phi, \tilde{w}) \phi^{2}\right)_{x x}=0, \\
\phi(x, 0)=\phi_{0}(x)=w_{0}(x)-\tilde{w}(x, 0) .
\end{array}\right.
$$

Here

$$
\begin{gathered}
\psi(\tilde{w})=-\gamma \tilde{w}^{-(\gamma+1)} \\
g(\phi, \tilde{w}) \phi^{2}=(\phi+\tilde{w})^{-\gamma}-\tilde{w}^{-\gamma}-\psi(\tilde{w}) \phi .
\end{gathered}
$$

Now let $F=-\psi(\tilde{w}) \phi$; the corresponding problem on $F$ is given by

$$
\left\{\begin{array}{c}
F_{t}+a(x) \psi(\tilde{w}) F_{x x}-\psi(\tilde{w})\left(a-a_{1}\right)\left(\tilde{w}^{-\gamma}\right)_{x x} \\
-\psi_{1}(\tilde{w}) F \tilde{w}_{t}-a \psi(\tilde{w})\left(f F^{2}\right)_{x x}=0 \\
F(x, 0)=F_{0}(x)=-\psi(\tilde{w}(x, 0)) \phi_{0}(x)
\end{array}\right.
$$

where

$$
-\psi_{1}(\tilde{w}) F=\psi^{\prime}(\tilde{w}) \phi, f F^{2}=g \phi^{2} .
$$

We will establish the global existence and large time behavior, for the solution $F$ to $(2.5)$, in the Banach space $X(0, T)$ defined for all $T>0$ by

$$
X(0, t)=\left\{F \in C^{0}\left([0, t] ; H^{2}\right), \quad 0 \leq t \leq T\right\}
$$


and equipped with the norm

$$
N^{2}(t)=\sup _{0 \leq \tau \leq t}\|F(\tau)\|_{H^{2}}^{2}
$$

The main result of this subsection is the following theorem.

TheOREM 2.2. Assume that $F_{0}(x)$ and $s(x)=s_{0}(x)$ are $C^{2}$ functions such that $F_{0} \in H^{2}(R) \cap L^{1}(R)$ and

$$
x(s(x)-\bar{s}) \in L^{1}(R) .
$$

Then there exist constants $\varepsilon_{0}>0$ and $\delta>0$ such that if $\left|w_{+}-w_{-}\right| \leq \delta$ and $\left\|F_{0}\right\|_{H^{2}} \leq$ $\varepsilon_{0}$, then (2.5) has a unique global smooth solution $F$ satisfying

$$
\sum_{j=0}^{2} w_{j+1}(t)\left\|\partial_{x}^{j} F(\cdot, t)\right\|^{2}+\int_{0}^{t} \sum_{j=1}^{3} w_{j}(\tau)\left\|\partial_{x}^{j} F(\cdot, \tau)\right\|^{2} d \tau \leq C,
$$

where the weight functions $w_{j}(t)$ are given by

$$
w_{1}(t)=(1+t)^{\frac{1}{2}}(1+\log (1+t))^{-k}, w_{j}(t)=(1+t)^{j-1} w_{1}(t)
$$

for $j, k>1$.

Remark 3. (a) The condition (2.6) plays an important role in our proof of Theorem 2.2 (see Lemmas 2.3-2.7, 2.9-2.10 below). This condition enables us to bound the $L^{1}$-norm of $F$ for all time. In [7], $s(x)-\bar{s}$ is assumed to be compact support besides the technical condition V; our condition (2.6) is much weaker. In fact, (2.6) asks only some decay properties on $s(x)-\bar{s}$ as $x \rightarrow \pm \infty$.

(b) The condition (2.6) can be replaced by the weaker one such as

$$
|x|^{\beta}(s(x)-\bar{s}) \in L^{1}(R)
$$

for some $\beta>0$. This is clear following our proof.

(c) In general, we could not bound the $L^{1}$-norm of $F$ for all time without the conditions on the decay properties of $s(x)-\bar{s}$ as $x \rightarrow \pm \infty$ such as $\left(2.6^{\prime}\right)$. One cannot even bound the total mass of $F$ uniformly in time under the condition $s(x)-\bar{s} \in L^{1}$. From this point of view, $\left(2.6^{\prime}\right)$ is optimal.

The local existence and uniqueness of the solution to $(2.5)$ in $X(0, T)$ is standard, so to get the global existence, we will prove uniform estimates on the solution of (2.5). Hence, from now on, we assume the local existence in $X(0, T)$ for some $T>0$.

The following $L^{1}$-estimate follows from the standard contraction property of the porous media type equation and will play a fundamental role in the rest of this section.

LEMma 2.3. Under the conditions of Theorem 2.2, as long as the solution exists in $X(0, T)$, there exist positive constants $C_{1}$ and $C_{2}$, such that

$$
\|\phi(\cdot, t)\|_{L^{1}} \leq C_{1}\|F(\cdot, t)\|_{L^{1}} \leq C_{2}\left(\left\|\phi_{0}\right\|_{L^{1}}+\delta\right) .
$$

Proof. We present here a formal argument which can easily be made rigorous by using any sequence approximating the sign function and passing into the limit by means of the Lebesgue dominated convergence theorem. Observe that $h=\operatorname{sign}(\phi)=$ $\operatorname{sign}(F)$. Let us multiply the equation in $(2.4)$ by $a^{-1} h$, then by integrating over 
$[0, t] \times(-\infty,+\infty)$, it follows that

$$
\begin{aligned}
& \int_{-\infty}^{+\infty} a^{-1}|\phi|(x, t) d x+\int_{0}^{t} \int_{-\infty}^{+\infty} \operatorname{sign}^{\prime}(F) F_{x}^{2} d x d \tau \\
& \leq C \int_{-\infty}^{+\infty} a^{-1}\left|\phi_{0}\right|(x) d x+C\left|\int_{0}^{t} \int_{-\infty}^{+\infty}\left(a-a_{1}\right) \tilde{w}_{t} \operatorname{sign}(F) d x d \tau\right| \\
& +\left|\int_{0}^{t} \int_{-\infty}^{+\infty}\left(f F^{2}\right)_{x} F_{x} \operatorname{sign}^{\prime}(F) d x d \tau\right| \\
& \leq C\left(\left\|\phi_{0}\right\|_{L^{1}}+\delta\right) .
\end{aligned}
$$

Here, we have used the following facts:

$$
\begin{aligned}
& \quad\left|\int_{0}^{t} \int_{-\infty}^{+\infty}\left(a-a_{1}\right) \tilde{w}_{t} \operatorname{sign}(F) d x d \tau\right| \\
& \leq C \int_{0}^{t} \int_{-\infty}^{+\infty}|s-\bar{s}|\left|\tilde{w}_{t}\right| d x d \tau \\
& \quad \leq C \int_{0}^{t} \int_{-\infty}^{+\infty}(1+t)^{-\frac{3}{2}}|x(s-\bar{s})|\left|\tilde{w}^{\prime}(\eta)\right| d x d \tau \\
& \quad \leq C \delta \\
& \int_{0}^{t} \int_{-\infty}^{+\infty}\left(f F^{2}\right)_{x} F_{x} \operatorname{sign}^{\prime}(F) d x d \tau \\
& =\int_{0}^{t} \int_{-\infty}^{+\infty} F_{x}\left(2 f F_{x}+f_{F} F F_{x}+f_{\tilde{w}} F \tilde{w}_{x}\right) F \delta_{\{F=0\}} d x d \tau \\
& =0 .
\end{aligned}
$$

Hence (2.8) gives the proof of (2.7).

With the help of Lemma 2.3, we can make the energy estimates on $F$.

Lemma 2.4. Under the hypotheses of Theorem 2.2, there exists $\varepsilon_{*}>0$ such that if $0<\varepsilon<\varepsilon_{*}$ and $N(T) \leq \varepsilon$, then we have

$$
\|F(\cdot, t)\|^{2}+\int_{0}^{t}\left\|F_{x}(\cdot, \tau)\right\|^{2} d \tau \leq C\left(\left\|F_{0}\right\|^{2}+\delta\right)
$$

for $0 \leq t \leq T$.

Proof. Let us multiply (2.4) by $a^{-1} F$ and integrate the result over $[0, t] \times$ $(-\infty,+\infty)$; we then get

$$
\begin{aligned}
& \int_{-\infty}^{+\infty} \frac{1}{2} a^{-1} F \phi(x, t) d x+\int_{0}^{t} \int_{-\infty}^{+\infty} F_{x}^{2} d x d \tau \\
& \leq \int_{-\infty}^{+\infty} \frac{1}{2} a^{-1} F_{0} \phi_{0} d x+\left|\int_{0}^{t} \int_{-\infty}^{+\infty} a^{-1}\left(a-a_{1}\right)\left(\tilde{w}^{-\gamma}\right)_{x x} F d x d \tau\right| \\
& +\left|\int_{0}^{t} \int_{-\infty}^{+\infty} \frac{1}{2} a^{-1} \psi_{2}(\tilde{w}) F^{2} \tilde{w}_{t} d x d \tau\right|+\left|\int_{0}^{t} \int_{-\infty}^{+\infty}\left(f F^{2}\right)_{x} F_{x} d x d \tau\right| \\
& \equiv \int_{-\infty}^{+\infty} \frac{1}{2} a^{-1} F_{0} \phi_{0} d x+I_{1}+I_{2}+I_{3}
\end{aligned}
$$


with $\psi_{2}(\tilde{w}) F^{2}=\phi^{2} \psi^{\prime}(\tilde{w})$.

We use $I_{1}, I_{2}$, and $I_{3}$ step-by-step as follows:

$$
\begin{aligned}
& I_{1}=\left|\int_{0}^{t} \int_{-\infty}^{+\infty} a^{-1}\left(a-a_{1}\right)(\tilde{w})_{x x}^{-\gamma} F d x d \tau\right| \\
& \leq C \delta \varepsilon \int_{0}^{t}(1+\tau)^{-\frac{3}{2}}\|x(s-\bar{s})\|_{L^{1}} d \tau \\
& \leq C \delta \varepsilon, \\
& I_{2}=\left|\int_{0}^{t} \int_{-\infty}^{+\infty} \frac{1}{2} a^{-1} \psi_{2}(\tilde{w}) F^{2} \tilde{w}_{t} d x d \tau\right| \\
& \leq C \int_{0}^{t}\|F\|_{L^{\infty}}\left\|\tilde{w}_{t}\right\|_{L^{\infty}}\|F\|_{L^{1}} d x d \tau \\
& \leq C \delta \int_{0}^{t}\|F\|^{\frac{1}{2}}\left\|F_{x}\right\|^{\frac{1}{2}}(1+\tau)^{-1} d \tau \\
& \leq C \delta\left(\int_{0}^{t}\|F\|^{2}\left\|F_{x}\right\|^{2} d \tau+\int_{0}^{t}(1+\tau)^{-\frac{4}{3}} d \tau\right) \\
& \leq C \delta\left(1+\varepsilon^{2} \int_{0}^{t}\left\|F_{x}\right\|^{2} d \tau\right), \\
& I_{3}=\left|\int_{0}^{t} \int_{-\infty}^{+\infty}\left(f F^{2}\right)_{x} F_{x} d x d \tau\right| \\
& \leq\left(\frac{1}{2}+C \varepsilon\right) \int_{0}^{t}\left\|F_{x}\right\|^{2} d \tau+C \delta^{2} \int_{0}^{t}\|F\|_{L^{\infty}}^{4} d \tau \\
& \leq\left(\frac{1}{2}+C \varepsilon\right) \int_{0}^{t}\left\|F_{x}\right\|^{2} d \tau+C \delta^{2} \int_{0}^{t}\|F\|^{2}\left\|F_{x}\right\|^{2} d \tau \\
& \leq\left(\frac{1}{2}+C \varepsilon\right) \int_{0}^{t}\left\|F_{x}\right\|^{2} d \tau \text {. }
\end{aligned}
$$

Due to the smallness of $\delta$ and $\varepsilon$, we conclude from (2.12)-(2.15) that

$$
\|F(\cdot, t)\|^{2}+\int_{0}^{t}\left\|F_{x}(\cdot, \tau)\right\|^{2} d \tau \leq C\left(\left\|F_{0}\right\|^{2}+\delta\right),
$$

which completes the proof of Lemma 2.4.

For higher order estimates, we use the problem (2.5) to obtain the following results.

LEMma 2.5. Under the same conditions of Lemma 2.4, we have

$$
\left\|F_{x}(\cdot, t)\right\|^{2}+\int_{0}^{t}\left\|F_{x x}(\cdot, \tau)\right\|^{2} d \tau \leq C\left(\left\|F_{0}\right\|_{H^{1}}^{2}+\delta\right) .
$$


Proof. Let us multiply the equation in (2.5) by $F_{x x}$, then

$$
\begin{gathered}
\int_{-\infty}^{+\infty} F_{x}^{2}(x, t) d x+\int_{0}^{t} \int_{-\infty}^{+\infty} F_{x x}^{2}(x, \tau) d x d \tau \\
\leq C\left(\left\|F_{0 x}\right\|^{2}+\left|\int_{0}^{t} \int_{-\infty}^{+\infty} \tilde{w}_{t} F_{x x} d x d \tau\right|\right. \\
\left.\quad+\left|\int_{0}^{t} \int_{-\infty}^{+\infty}\left(f F^{2}\right)_{x x} F_{x x} d x d \tau\right|\right)
\end{gathered}
$$

which implies, with the help of the Cauchy-Schwarz inequality and Lemma 2.1, that

$$
\begin{aligned}
& \int_{-\infty}^{+\infty} F_{x}^{2}(x, t) d x+\int_{0}^{t} \int_{-\infty}^{+\infty} F_{x x}^{2}(x, \tau) d x d \tau \\
& \leq C\left(\left\|F_{0 x}\right\|^{2}+\delta^{2}\right)+C \int_{0}^{t} \int_{-\infty}^{+\infty}\left(f F^{2}\right)_{x x}^{2} d x d \tau .
\end{aligned}
$$

We bound the last term in (2.19) as follows:

$$
\begin{aligned}
& \int_{0}^{t} \int_{-\infty}^{+\infty}\left(f F^{2}\right)_{x x}^{2} d x d \tau \\
& \leq C \int_{0}^{t} \int_{-\infty}^{+\infty}\left[\left(|F|+\left|F_{x}\right|+\left|w_{x}\right|\right)^{2} F_{x}^{2}+F^{2} F_{x x}^{2}+F^{4}\left(\tilde{w}_{x x}^{2}+\tilde{w}_{x}^{4}\right)\right] d x d \tau \\
& \leq C \varepsilon^{2} \delta^{2}+C \varepsilon \int_{0}^{t} \int_{-\infty}^{+\infty} F_{x x}^{2}(\tau, x) d x d \tau .
\end{aligned}
$$

Then, by (2.19)-(2.20) and the estimates in Lemma 2.4, we get (2.17).

We now turn to the third order estimates. For this purpose, we differentiate the equation in (2.5) with respect to $x$

$$
\begin{aligned}
& F_{t x}+a \psi(\tilde{w}) F_{x x x}+(a \psi(\tilde{w}))_{x} F_{x x}-\left(\psi(\tilde{w})\left(a-a_{1}\right)\left(\tilde{w}^{-\gamma}\right)_{x x}\right)_{x} \\
& \quad+\left(\psi_{1}(\tilde{w}) F \tilde{w}_{t}\right)_{x}-\left(a \psi(\tilde{w})\left(f F^{2}\right)_{x x}\right)_{x}=0 .
\end{aligned}
$$

Multiplying (2.21) by $F_{x x x}$ and then integrating it over $[0, t] \times(-\infty,+\infty)$, one has

$$
\begin{aligned}
& \quad \int_{-\infty}^{+\infty} F_{x x}^{2}(\cdot, t) d x+\int_{0}^{t} \int_{-\infty}^{+\infty} F_{x x x}^{2}(\tau, x) d x d \tau \\
& \leq C\left(\left\|F_{0 x x}\right\|^{2}+\int_{0}^{t} \int_{-\infty}^{+\infty}\left((a \psi)_{x} F_{x x}\right)^{2} d x d \tau+\int_{0}^{t} \int_{-\infty}^{+\infty}\left(\psi_{1} \tilde{w} F \tilde{w}_{t}\right)_{x}^{2} d x d \tau\right. \\
& \quad+\int_{0}^{t} \int_{-\infty}^{+\infty}\left[\left(\left(\psi(\tilde{w})\left(a-a_{1}\right)\left(\tilde{w}^{-\gamma}\right)_{x x}\right)_{x}^{2}+\left(a \psi(\tilde{w})\left(f F^{2}\right)_{x x}\right)_{x}^{2}\right] d x d \tau\right) \\
& \leq C\left(\left\|F_{0}\right\|_{H^{2}}^{2}+\delta^{2}\right)+C \varepsilon \int_{0}^{t} \int_{-\infty}^{+\infty} F_{x x x}^{2}(\tau, x) d x d \tau
\end{aligned}
$$

which can be summarized as follows.

Lemma 2.6. Under the same conditions as Lemma 2.4, one has

$$
\left\|F_{x x}(\cdot, t)\right\|^{2}+\int_{0}^{t}\left\|F_{x x x}(\cdot, \tau)\right\|^{2} d \tau \leq C\left(\left\|F_{0}\right\|_{H^{2}}^{2}+\delta\right) .
$$


From Lemma 2.4-2.6, we can conclude the following.

LEMma 2.7. Under the same conditions as Theorem 2.2, there exists $\varepsilon_{*}>0$ such that if $0<\varepsilon<\varepsilon_{*}$ and $N(T) \leq \varepsilon$, then it follows that

$$
\|F(t)\|_{H^{2}}^{2}+\int_{0}^{t}\left(\left\|F_{t}\right\|_{H^{1}}^{2}+\left\|F_{x}\right\|_{H^{2}}^{2}\right)(\tau) d \tau \leq C_{0}\left(\left\|F_{0}\right\|_{H^{2}}^{2}+\delta\right)
$$

for all $0 \leq t \leq T$, where $C_{0}$ is a positive constant independent of $t$.

With the help of the previous lemmas we obtain the global existence of the solution $F(x, t)$ to $(2.5)$.

THEOREM 2.8. Under the same conditions as Theorem 2.2, (2.5) has a unique global smooth solution $F(x, t)$ which tends to zero uniformly in $H^{1}$ as $t$ goes to infinity.

Proof. We choose $\delta, \varepsilon_{0}$, and $\varepsilon$ small such that $C_{0}\left(\varepsilon_{0}^{2}+\delta\right) \leq \varepsilon^{3}, \varepsilon \leq \frac{1}{4}$ so that all the previous arguments are valid. Then, due to the local results, there exists a positive $t_{1}$ such that the solution $F(x, t)$ exists in $(-\infty,+\infty) \times\left[0, t_{1}\right]$ and satisfies

$$
N(t)^{2} \leq 4 N^{2}(0) \text { for all } t \in\left[0, t_{1}\right] .
$$

We can apply the $L^{1}$-estimate for $F$ of Lemma 2.3 and then Lemma 2.7 in $0 \leq t \leq t_{1}$. Therefore, it follows that

$$
N^{2}(t) \leq C_{0}\left(\left\|F_{0}\right\|_{H^{2}}^{2}+\delta\right) \leq \varepsilon^{3} \text { for all } t \in\left[0, t_{1}\right] .
$$

By iterating the above procedure, a standard continuity argument allows us to establish the global existence in time for the solution to (2.5).

Now, from Lemma 2.7 and the above argument, we have

$$
\|F(t)\|_{H^{2}}^{2}+\int_{0}^{t}\left(\left\|F_{x}\right\|_{H^{2}}^{2}+\left\|F_{t}\right\|_{H^{1}}^{2}\right)(\tau) d \tau \leq C_{0}\left(\left\|F_{0}\right\|_{H^{2}}^{2}+\delta\right) \text { for all } t>0 .
$$

From (2.24) we know that

$$
\left\|F_{x}(t)\right\|^{2}+\int_{0}^{+\infty}\left|\frac{d}{d t}\left\|F_{x}(t)\right\|^{2}\right| d t \leq C
$$

which implies

$$
\lim _{t \rightarrow+\infty}\left\|F_{x}(t)\right\|^{2}=0
$$

Then, the Sobolev inequality implies

$$
\begin{aligned}
\lim _{t \rightarrow+\infty}\|F(t)\|^{2} \leq & \lim _{t \rightarrow+\infty}\|F(t)\|_{L^{\infty}}\|F(t)\|_{L^{1}} \\
& =O(1) \lim _{t \rightarrow+\infty}\left\|F_{x}(t)\right\|^{\frac{1}{2}} \\
& =0
\end{aligned}
$$

which completes the proof of this theorem.

By using the weighted energy method, we can prove the following decay estimates.

LEMmA 2.9. Let $F$ be the solution to (2.5) obtained in Theorem 2.8, then

$$
\begin{aligned}
& w_{1}(t)\|F(t)\|^{2}+w_{2}(t)\left\|F_{x}(t)\right\|^{2} \\
& +\int_{0}^{t}\left(w_{1}(\tau)\left\|F_{x}(\tau)\right\|^{2}+w_{2}(\tau)\left\|F_{x x}(\tau)\right\|^{2}\right) d \tau \leq C .
\end{aligned}
$$


Proof. Let us multiply (2.5) by $w_{2}(t) F_{x x}$, then we get

$$
\begin{aligned}
& \left(\frac{1}{2} w_{2}(t) F_{x}^{2}\right)_{t}-a \psi(\tilde{w}) w_{2}(t) F_{x x}^{2}-\frac{1}{2} w_{2}^{\prime}(t) F_{x}^{2}-\psi_{1}(\tilde{w}) F F_{x x} \tilde{w}_{t} w_{2}(t) \\
& =-w_{2}(t) \psi(\tilde{w})\left(a-a_{1}\right)\left(\tilde{w}^{-\gamma}\right)_{x x} F_{x x}-a \psi(\tilde{w})\left(f F^{2}\right)_{x x} F_{x x} w_{2}(t)+(\cdots)_{x},
\end{aligned}
$$

where $(\cdots)_{x}$ denotes the term which does not need to be computed explicitly since it will disappear by integrating in $x$. Then one has

$$
\begin{aligned}
& w_{2}(t)\left\|F_{x}(\cdot, t)\right\|^{2}+\int_{0}^{t} w_{2}(\tau)\left\|F_{x x}(\cdot, \tau)\right\|^{2} d \tau \\
& \leq C_{1}\left(\left\|F_{0 x}\right\|^{2}+\left|\int_{0}^{t} \int_{-\infty}^{+\infty} w_{2}^{\prime}(\tau) F_{x}^{2} d x d \tau\right|\right. \\
& +\left|\int_{0}^{t} \int_{-\infty}^{+\infty} \tilde{w}_{t}^{2} w_{2}(\tau)\left(a-a_{1}\right)^{2} d x d \tau\right| \\
& \left.+\left|\int_{0}^{t} \int_{-\infty}^{+\infty} F^{2} \tilde{w}_{t}^{2} w_{2}(\tau) d x d \tau\right|+\int_{0}^{t} \int_{-\infty}^{+\infty} w_{2}(\tau)\left(f F^{2}\right)_{x x}^{2} d x d \tau\right) \\
& \equiv C_{1}\left(\left\|F_{0 x}\right\|^{2}+J_{1}+J_{2}+J_{3}+J_{4}\right) .
\end{aligned}
$$

On the other hand, if we multiply $(2.4)$ by $a^{-1} w_{1}(t) F$, we get

$$
\begin{aligned}
& \left(\frac{1}{2} F \phi a^{-1} w_{1}(t)\right)_{t}+w_{1}(t) F_{x}^{2}-\frac{1}{2} w_{1}^{\prime}(t) a^{-1} \psi_{1}(\tilde{w}) F^{2} \\
& =\frac{1}{2} a^{-1} w_{1}(t) F^{2} \tilde{w}_{t}-a^{-1} w_{1}(t)\left(a-a_{1}\right) F\left(\tilde{w}^{-\gamma}\right)_{x x} \\
& +w_{1}(t) F_{x}\left(f F^{2}\right)_{x}+(\cdots)_{x},
\end{aligned}
$$

which, integrated on $[0, t] \times(-\infty,+\infty)$, yields

$$
\begin{aligned}
& w_{1}(t)\|F(\cdot, t)\|^{2}+\int_{0}^{t} w_{1}(t)\left\|F_{x}(\tau)\right\|^{2} d \tau \\
& \leq C_{2}\left(\left\|F_{0}\right\|^{2}+\left|\int_{0}^{t} \int_{-\infty}^{+\infty} w_{1}^{\prime}(\tau) F^{2} d x d \tau\right|\right. \\
& +\left|\int_{0}^{t} \int_{-\infty}^{+\infty} w_{1}(\tau) F^{2} \tilde{w}_{t} d x d \tau\right|+\left|\int_{0}^{t} \int_{-\infty}^{+\infty} w_{1}(\tau) \tilde{w}_{t} F\left(a-a_{1}\right) d x d \tau\right| \\
& \left.+\left|\int_{0}^{t} \int_{-\infty}^{+\infty} w_{1}(\tau) F_{x}\left(f F^{2}\right)_{x} d x d \tau\right|\right) \\
& \equiv C_{2}\left(\left\|F_{0}\right\|^{2}+J_{5}+J_{6}+J_{7}+J_{8}\right) .
\end{aligned}
$$

By calculating $K \times(2.29)+(2.27)$ with a $K>0$ to be determined later, we have

$$
\begin{aligned}
& K w_{1}(t)\|F(t)\|^{2}+w_{2}(t)\left\|F_{x}(t)\right\|^{2} \\
& +\int_{0}^{t}\left(K w_{1}(\tau)\left\|F_{x}(\tau)\right\|^{2}+w_{2}(\tau)\left\|F_{x x}(\tau)\right\|^{2}\right) d \tau \\
& \quad \leq\left(C_{1}\left\|F_{0 x}\right\|^{2}+K C_{2}\left\|F_{0}\right\|^{2}\right) \\
& \quad+C_{1}\left(J_{1}+J_{2}+J_{3}+J_{4}\right)+K C_{2}\left(J_{5}+J_{6}+J_{7}+J_{8}\right) .
\end{aligned}
$$


The following inequalities will be used to estimate the terms on $J_{i}(i=1, \ldots, 8)$ :

$$
\begin{aligned}
\left|w_{1}^{\prime}(t)\right|= & \left|\frac{1}{2}(1+t)^{-1} w_{1}(t)-k(1+\log (1+t))^{-1}(1+t)^{-1} w_{1}(t)\right| \\
\leq & C(1+t)^{-1} w_{1}(t), \\
& \left|w_{2}^{\prime}(t)\right| \leq C(1+t)^{-1} w_{2}(t)=C w_{1}(t) .
\end{aligned}
$$

Then, by choosing $K$ large enough, one has

$$
\begin{gathered}
C_{1} J_{1} \leq \frac{1}{2} K \int_{0}^{t} w_{1}(\tau)\left\|F_{x}(\tau)\right\|^{2} d \tau, \\
J_{2} \leq C \delta^{2} \int_{0}^{t}(1+\tau)^{-3} w_{2}(\tau) d \tau \leq C \delta^{2} .
\end{gathered}
$$

To estimate $J_{3}$, observe that the following inequality on $F$ holds:

$$
\|F\|_{L^{\infty}} \leq C\left\|F_{x}\right\|^{\frac{2}{3}}
$$

since

$$
\begin{aligned}
\|F\|_{L^{\infty}} & \leq C\|F\|^{\frac{1}{2}}\left\|F_{x}\right\|^{\frac{1}{2}} \\
& \leq C\|F\|_{L^{\infty}}^{\frac{1}{4}}\left\|F_{x}\right\|^{\frac{1}{2}}\|F\|_{L^{1}}^{\frac{1}{4}} .
\end{aligned}
$$

Then we see that

$$
\begin{aligned}
C_{1} J_{3} & =C_{1}\left|\int_{0}^{t} \int_{-\infty}^{+\infty} F^{2} \tilde{w}_{t}^{2} w_{2}(\tau) d x d \tau\right| \\
& \leq C \int_{0}^{t} w_{2}(\tau)\left\|\tilde{w}_{t}\right\|_{L^{\infty}}\|F\|_{L^{\infty}}\|F\|_{L^{1}} d \tau \\
& \leq C \delta^{2} \int_{0}^{t}(1+\tau)^{-2} w_{2}(\tau)\left\|F_{x}\right\|^{\frac{2}{3}} d \tau \\
& \leq C \delta^{2}+\frac{1}{4} K \int_{0}^{t} w_{1}(\tau)\left\|F_{x}(\cdot, \tau)\right\|^{2} d \tau
\end{aligned}
$$

Similarly, we can estimate $J_{5}, J_{6}$, and $J_{7}$ as follows:

$$
\begin{aligned}
C_{2} K J_{5} & =C_{2} K\left|\int_{0}^{t} \int_{-\infty}^{+\infty} w_{1}^{\prime}(\tau) F^{2} d x d \tau\right| \\
& \leq C K \int_{0}^{t}(1+\tau)^{-1} w_{1}(\tau)\left\|F_{x}\right\|^{\frac{2}{3}} d \tau \\
& \leq C K+\frac{1}{8} K \int_{0}^{t} w_{1}(\tau)\left\|F_{x}(\cdot, \tau)\right\|^{2} d \tau,
\end{aligned}
$$




$$
\begin{aligned}
C_{2} K J_{6} & =C_{2} K\left|\int_{0}^{t} \int_{-\infty}^{+\infty} w_{1}(\tau) F^{2} \tilde{w}_{t} d x d \tau\right| \\
& \leq C K \delta+\frac{1}{16} K \int_{0}^{t} w_{1}(\tau)\left\|F_{x}(\cdot, \tau)\right\|^{2} d \tau, \\
C_{2} K J_{7} & =C_{2} K\left|\int_{0}^{t} \int_{-\infty}^{+\infty} w_{1}(\tau) \tilde{w}_{t} F\left(a-a_{1}\right) d x d \tau\right| \\
\leq & C K \delta+\frac{1}{32} K \int_{0}^{t} w_{1}(\tau)\left\|F_{x}(\cdot, \tau)\right\|^{2} d \tau .
\end{aligned}
$$

To estimate $J_{8}$, we have

$$
\begin{aligned}
& C_{2} K J_{8} \\
& =C_{2} K\left|\int_{0}^{t} \int_{-\infty}^{+\infty} w_{1}(\tau) F_{x}\left(f F^{2}\right)_{x} d x d \tau\right| \\
& \leq C\left|\int_{0}^{t} \int_{-\infty}^{+\infty} w_{1}(\tau) F_{x}\left(2 f F F_{x}+f_{F} F^{2} F_{x}+f_{\tilde{w}} \tilde{w}_{x} F^{2}\right) d x d \tau\right| \\
& \leq C \varepsilon K \int_{0}^{t} w_{1}(\tau)\left\|F_{x}(\cdot, \tau)\right\|^{2} d \tau+C K \int_{0}^{t} \int_{-\infty}^{+\infty} w_{1}(\tau) \tilde{w}_{x}^{2} F^{2} d x d \tau \\
& \leq C K(\delta+\varepsilon) \int_{0}^{t} w_{1}(\tau)\left\|F_{x}(\cdot, \tau)\right\|^{2} d \tau+C K \delta .
\end{aligned}
$$

We now deal with the term $J_{4}$. Noting that

$$
\begin{aligned}
\left(f F^{2}\right)_{x x}= & \left(2 F F_{x} f+f_{F} F^{2} F_{x}+f_{\tilde{w}} \tilde{w}_{x} F^{2}\right)_{x} \\
= & \left(2 f F+f_{F} F^{2}\right) F_{x x}+\left(2 f+4 f_{F} F+f_{F F} F^{2}\right) F_{x}^{2} \\
& \quad+\left(4 f_{\tilde{w}} F+2 f_{F \tilde{w}} F^{2}\right) F_{x} \tilde{w}_{x}+\left(f_{\tilde{w}} \tilde{w}_{x x}+f_{\tilde{w} \tilde{w}} \tilde{w}_{x}^{2}\right) F^{2},
\end{aligned}
$$

we have

$$
\begin{aligned}
J_{4}=\left|\int_{0}^{t} \int_{-\infty}^{+\infty} w_{2}(\tau)\left(f F^{2}\right)_{x x}^{2} d x d \tau\right| \\
\leq C \varepsilon \int_{0}^{t} w_{2}(\tau)\left\|F_{x x}(\cdot, \tau)\right\|^{2} d \tau+C \int_{0}^{t} \int_{-\infty}^{+\infty} F_{x}^{4} w_{2}(\tau) d x d \tau \\
\quad+C\left|\int_{0}^{t} \int_{-\infty}^{+\infty} w_{2}(\tau) F_{x}^{2} \tilde{w}_{x}^{2} F d x d \tau\right| \\
\quad+C \int_{0}^{t} \int_{-\infty}^{+\infty} w_{2}(\tau) F^{4}\left(\tilde{w}_{x x}^{2}+\tilde{w}_{x}^{4}\right) d x d \tau \\
\equiv C \varepsilon \int_{0}^{t} w_{2}(\tau)\left\|F_{x x}(\cdot, \tau)\right\|^{2} d \tau+C\left(J_{9}+J_{10}+J_{11}\right) .
\end{aligned}
$$


We can estimate $J_{10}$ and $J_{11}$ in the following way:

$$
\begin{gathered}
J_{10}=\left|\int_{0}^{t} \int_{-\infty}^{+\infty} w_{2}(\tau) F_{x}^{2} \tilde{w}_{x}^{2} F d x d \tau\right| \\
\leq C \varepsilon \delta^{2} \int_{0}^{t} w_{1}(\tau)\left\|F_{x}(\cdot, \tau)\right\|^{2} d \tau, \\
J_{11}=\int_{0}^{t} \int_{-\infty}^{+\infty} w_{2}(\tau) F^{4}\left(\tilde{w}_{x x}^{2}+\tilde{w}_{x}^{4}\right) d x d \tau \\
\leq C \delta^{2} \varepsilon^{2} \int_{0}^{t} w_{1}(\tau)\left\|F_{x}(\cdot, \tau)\right\|^{2} d \tau,
\end{gathered}
$$

while $J_{9}$ can be bounded as follows:

$$
\begin{aligned}
J_{9} & =\int_{0}^{t} \int_{-\infty}^{+\infty} F_{x}^{4} w_{2}(\tau) d x d \tau \\
& \leq C \varepsilon^{2} \int_{0}^{t} w_{2}(\tau)\left\|F_{x x}\right\|^{2} d \tau+C \int_{0}^{t} w_{2}(\tau)\left\|F_{x}(\tau)\right\|^{2}\left\|F_{x}(\tau)\right\|^{2} d \tau .
\end{aligned}
$$

Due to the smallness of $\delta$ and $\varepsilon$, by choosing $K$ large enough, we deduce

$$
\begin{aligned}
& w_{1}(t)\|F(t)\|^{2}+w_{2}(t)\left\|F_{x}(t)\right\|^{2} \\
& +\int_{0}^{t}\left(w_{1}(\tau)\left\|F_{x}(\tau)\right\|^{2}+w_{2}(\tau)\left\|F_{x x}(\tau)\right\|^{2}\right) d \tau \\
& \leq C\left(1+\int_{0}^{t} w_{2}(\tau)\left\|F_{x}(\tau)\right\|^{2}\left\|F_{x}(\tau)\right\|^{2} d \tau\right) .
\end{aligned}
$$

Therefore, from (2.45), it follows that

$$
w_{2}(t)\left\|F_{x}(t)\right\|^{2} \leq C\left(1+\int_{0}^{t} w_{2}(\tau)\left\|F_{x}(\tau)\right\|^{2}\left\|F_{x}(\tau)\right\|^{2} d \tau\right),
$$

which implies, with the help of Gronwall inequality, that

$$
w_{2}(t)\left\|F_{x}(t)\right\|^{2} \leq C
$$

and

$$
\int_{0}^{t} w_{2}(\tau)\left\|F_{x}(\tau)\right\|^{2}\left\|F_{x}(\tau)\right\|^{2} d \tau \leq C .
$$

Hence, (2.45) and (2.46) give the proof of this lemma.

The following lemma contains the decay rates for the derivatives of $F$, which will be useful in the next section.

Lemma 2.10. The solution F to (2.5), obtained in Theorem 2.8, satisfies

$$
\begin{aligned}
w_{3}(t)\left\|F_{t}(t)\right\|^{2} & +w_{4}(t)\left\|F_{t x}\right\|^{2}+\int_{0}^{t}\left(w_{3}(\tau)\left\|F_{t x}(\tau)\right\|^{2}+w_{4}(\tau)\left\|F_{t x x}(\tau)\right\|^{2}\right) d \tau \\
& \leq C \delta \\
\left\|F_{t}\right\|_{L^{\infty}} & \leq C w_{3}(t)^{-\frac{1}{4}} w_{4}(t)^{-\frac{1}{4}} .
\end{aligned}
$$


Proof. It is sufficient to prove (2.47), since the estimate for $\left\|F_{t}\right\|_{L^{\infty}}$ can be derived from (2.47) by using Sobolev inequality.

Let us differentiate $(2.5)_{1}$ in $t$, then

$$
\begin{aligned}
& F_{t t}+a \psi(\tilde{w}) F_{t x x}+a \psi^{\prime}(\tilde{w}) \tilde{w}_{t} F_{x x}-\left[\psi(\tilde{w})\left(a-a_{1}\right)\left(\tilde{w}^{-\gamma}\right)_{x x}\right]_{t} \\
& -\left(\psi_{1}(\tilde{w}) F \tilde{w}_{t}\right)_{t}-\left[a \psi(\tilde{w})\left(f F^{2}\right)_{x x}\right]_{t}=0 .
\end{aligned}
$$

If we multiply $(2.48)$ by $a^{-1} w_{3}(t) F_{t}$, we get

$$
\begin{aligned}
& \left(\frac{1}{2} a^{-1} w_{3}(t) F_{t}^{2}\right)_{t}-\psi(\tilde{w}) w_{3}(t) F_{t x}^{2}+\frac{1}{2} F_{t}^{2} \psi(\tilde{w})_{x x} w_{3}(t)-\frac{1}{2} F_{t}^{2} a^{-1} w_{3}^{\prime}(t) \\
& +\psi^{\prime}(\tilde{w}) \tilde{w}_{t} F_{x x} w_{3}(t) F_{t}-a^{-1}\left[\psi(\tilde{w})\left(a-a_{1}\right)\left(\tilde{w}^{-\gamma}\right)_{x x}\right]_{t} w_{3}(t) F_{t} \\
& -a^{-1}\left(\psi_{1}(\tilde{w}) F \tilde{w}_{t}\right)_{t} w_{3}(t) F_{t}-\left[\psi(\tilde{w})\left(f F^{2}\right)_{x x}\right]_{t} w_{3}(t) F_{t}+\{\cdots\}_{x}=0 .
\end{aligned}
$$

From the proof of Lemma 2.9 and $(2.5)_{1}$ it is clear that

$$
\begin{aligned}
& \int_{0}^{t} w_{2}(\tau)\left\|F_{t}(\cdot, \tau)\right\|^{2} d \tau \\
& \leq C\left(\int_{0}^{t} w_{2}(t)\left\|F_{x x}(\tau)\right\|^{2} d \tau+\int_{0}^{t} \int_{-\infty}^{+\infty}\left(a-a_{1}\right)^{2} \tilde{w}_{t}^{2} w_{2}(\tau) d x d \tau\right. \\
& \left.\quad+\int_{0}^{t} \int_{-\infty}^{+\infty} F^{2} \tilde{w}_{t}^{2} w_{2}(\tau) d x d \tau+\int_{0}^{t} \int_{-\infty}^{+\infty}\left(f F^{2}\right)_{x x}^{2} w_{2}(\tau) d x d \tau\right) \\
& \leq C
\end{aligned}
$$

Moreover, we have

$$
\begin{gathered}
a^{-1}\left(\psi_{1}(\tilde{w}) F \tilde{w}_{t}\right)_{t} w_{3}(t) F_{t}=O(1)\left[\tilde{w}_{t} w_{3}(t) F_{t}^{2}+\left(\tilde{w}_{t}^{2}+\tilde{w}_{t t}\right) w_{3}(t) F F_{t}\right], \\
a^{-1}\left[\psi(\tilde{w})\left(a-a_{1}\right)\left(\tilde{w}^{-\gamma}\right)_{x x}\right]_{t} w_{3}(t) F_{t}=O(1)\left(a-a_{1}\right)\left(\tilde{w}_{t}^{2}+\tilde{w}_{t t}\right) w_{3}(t) F_{t}, \\
{\left[\psi(\tilde{w})\left(f F^{2}\right)_{x x}\right]_{t} w_{3}(t) F_{t}=O(1) \tilde{w}_{t}\left(f F^{2}\right)_{x x} w_{3}(t) F_{t}-\psi(\tilde{w})\left(f F^{2}\right)_{x x t} w_{3}(t) F_{t} .}
\end{gathered}
$$

Now, integrating (2.49) and integrating by parts, we have

$$
\begin{aligned}
w_{3}(t)\left\|F_{t}(t)\right\|^{2}+\int_{0}^{t} w_{3}(\tau)\left\|F_{t x}(\tau)\right\|^{2} d \tau \\
\leq C+C \int_{0}^{t} \int_{-\infty}^{+\infty} F_{t}^{2} w_{2}(\tau) d x d \tau+\int_{0}^{t} \int_{-\infty}^{+\infty} \tilde{w}_{t} w_{3}(\tau) F_{x x}^{2} d x d \tau \\
\quad+\int_{0}^{t} \int_{-\infty}^{+\infty}\left[\left(a-a_{1}\right)^{2}+F^{2}\right]\left(\tilde{w}_{t}^{2}+\tilde{w}_{t t}\right)^{2} w_{3}(\tau)(1+\tau) d x d \tau \\
\quad+\int_{0}^{t} \int_{-\infty}^{+\infty} w_{2}(\tau)\left(f F^{2}\right)_{x x}^{2} d x d \tau+\left|\int_{0}^{t} \int_{-\infty}^{+\infty} \psi(\tilde{w})\left(f F^{2}\right)_{x x t} w_{3}(\tau) F_{t} d x d \tau\right| \\
\leq C\left(1+\left|\int_{0}^{t} \int_{-\infty}^{+\infty} w_{3}(\tau)\left(f F^{2}\right)_{x t}\left(F_{t x}+F_{t} \tilde{w}_{x}\right) d x d \tau\right|\right) .
\end{aligned}
$$


To bound the previous terms, we observe that

$$
\begin{aligned}
& \left|\int_{0}^{t} \int_{-\infty}^{+\infty} w_{3}(\tau)\left(f F^{2}\right)_{x t} F_{t} \tilde{w}_{x} d x d \tau\right| \\
& \leq C+C \int_{0}^{t} \int_{-\infty}^{+\infty} w_{3}(\tau)\left(f F^{2}\right)_{x t}^{2} d x d \tau
\end{aligned}
$$

and

$$
\begin{aligned}
& \left|\int_{0}^{t} \int_{-\infty}^{+\infty} w_{3}(\tau)\left(f F^{2}\right)_{x t} F_{t x} d x d \tau\right| \\
& \leq \varepsilon_{3} \int_{0}^{t} \int_{-\infty}^{+\infty} w_{3}(\tau) F_{t x}^{2} d x d \tau+C\left(\varepsilon_{3}\right) \int_{0}^{t} \int_{-\infty}^{+\infty} w_{3}(\tau)\left(f F^{2}\right)_{x t}^{2} d x d \tau
\end{aligned}
$$

Then choosing $\varepsilon_{3}$ sufficient small, we have

$$
\begin{aligned}
& w_{3}(t)\left\|F_{t}(t)\right\|^{2}+\int_{0}^{t} w_{3}(\tau)\left\|F_{t x}(\tau)\right\|^{2} d \tau \\
& \leq C\left(1+\int_{0}^{t} \int_{-\infty}^{+\infty} w_{3}(\tau)\left(f F^{2}\right)_{x t}^{2} d x d \tau\right) .
\end{aligned}
$$

Moreover, since

$$
\begin{aligned}
\left(f F^{2}\right)_{x t}^{2}= & {\left[2 f F F_{x}, f_{F} F^{2} F_{x}+f_{\tilde{w}} \tilde{w}_{x} F^{2}\right]_{t}^{2} } \\
= & O(1)\left(|F| F_{x t}^{2}+F_{t}^{2} F_{x}^{2}+\tilde{w}_{t}^{2} F_{x}^{2} F^{2}\right. \\
& \left.+F^{2} F_{t}^{2} \tilde{w}_{x}^{2}+\left(\tilde{w}_{t x}^{2}+\tilde{w}_{t}^{2} \tilde{w}_{x}^{2}\right) F^{4}\right),
\end{aligned}
$$

it follows that

$$
\begin{aligned}
& \left.\int_{0}^{t} \int_{-\infty}^{+\infty} w_{3}(\tau)\left(f F^{2}\right)_{x t}^{2} d x d \tau\right) \\
& \leq C\left(\varepsilon \int_{0}^{t} w_{3}(\tau)\left\|F_{t x}\right\|^{2} d \tau+A_{1}+A_{2}+A_{3}+A_{4}\right),
\end{aligned}
$$

where, as in the previous estimates we get

$$
\begin{gathered}
A_{1}=\int_{0}^{t} \int_{-\infty}^{+\infty} w_{3}(\tau) F_{t}^{2} F_{x}^{2} d x d \tau \leq C, \\
A_{2}=\int_{0}^{t} \int_{-\infty}^{+\infty} w_{3}(\tau) \tilde{w}_{t}^{2} F_{x}^{2} F^{2} d x d \tau \leq C \delta^{2} \varepsilon^{2}, \\
A_{3}=\int_{0}^{t} \int_{-\infty}^{+\infty} w_{3}(\tau) F^{2} F_{t}^{2} \tilde{w}_{x}^{2} d x d \tau \leq C \delta^{2} \varepsilon^{2},
\end{gathered}
$$

and

$$
A_{4}=\int_{0}^{t} \int_{-\infty}^{+\infty} w_{3}(\tau)\left(\tilde{w}_{t x}^{2}+\tilde{w}_{t}^{2} \tilde{w}_{x}^{2}\right) F^{4} d x d \tau \leq C \delta^{2} .
$$


Thus, we conclude from $(2.52)-(2.57)$ that

$$
w_{3}(t)\left\|F_{t}(t)\right\|^{2}+\int_{0}^{t} w_{3}(\tau)\left\|F_{t x}(\tau)\right\|^{2} d \tau \leq C,
$$

which completes the proof of the first part of (2.47).

Let us turn to the second part of (2.47). For this purpose, we multiply (2.48) by $w_{4}(t) F_{t x x}$. After similar calculations as before, by virtue of (2.58), we get

$$
\begin{aligned}
& w_{4}(t)\left\|F_{t x}(t)\right\|^{2}+\int_{0}^{t} w_{4}(\tau)\left\|F_{t x x}(\tau)\right\|^{2} d \tau \\
& \leq C\left(1+B_{1}+B_{2}+B_{3}+B_{4}\right),
\end{aligned}
$$

where

$$
\begin{gathered}
B_{1}=\int_{0}^{t} \int_{-\infty}^{+\infty} \tilde{w}_{t}^{2} w_{4}(\tau) F_{x x}^{2} d x d \tau \leq C \delta^{2}, \\
B_{2}=\int_{0}^{t} \int_{-\infty}^{+\infty}\left(\tilde{w}_{t}^{2}+\tilde{w}_{t t}\right)^{2} w_{4}(\tau)\left(a-a_{1}\right)^{2} d x d \tau \leq C \delta^{2}, \\
B_{3}=\int_{0}^{t} \int_{-\infty}^{+\infty} w_{4}(\tau)\left[\psi_{1}(\tilde{w}) F \tilde{w}_{t}\right]_{t}^{2} d x d \tau \leq C \delta^{2},
\end{gathered}
$$

and

$$
\begin{aligned}
B_{4} & =\int_{0}^{t} \int_{-\infty}^{+\infty} w_{4}(\tau)\left[\psi\left(\tilde{w}\left(f F^{2}\right)_{x x}\right]_{t}^{2} d x d \tau\right. \\
& \leq C \delta^{2}+C \int_{0}^{t} \int_{-\infty}^{+\infty} w_{4}(\tau)\left(f F^{2}\right)_{t x x}^{2} d x d \tau .
\end{aligned}
$$

Hence, one has

$$
\begin{aligned}
& w_{4}(t)\left\|F_{t x}(t)\right\|^{2}+\int_{0}^{t} w_{4}(\tau)\left\|F_{t x x}(\tau)\right\|^{2} d \tau \\
& \leq C\left(1+\int_{0}^{t} \int_{-\infty}^{+\infty} w_{4}(\tau)\left(f F^{2}\right)_{t x x}^{2} d x d \tau\right) .
\end{aligned}
$$

In order to bound the last term in (2.60), we use the following identity:

$$
\begin{aligned}
& \left(f F^{2}\right)_{t x x}^{2} \\
& =O(1)\left[F^{2} F_{t x x}^{2}+F_{x}^{2} F_{t x}^{2}+F^{2} \tilde{w}_{x}^{2} F_{t x}^{2}\right. \\
& \left.+\left(\tilde{w}_{x x}^{2}+\tilde{w}_{x}^{4}\right) F^{2} F_{t}^{2}+\tilde{w}_{t x}^{2} F^{2} F_{x}^{2}\right] \\
& +O(1)\left[\left(F_{t}^{2} F_{x x}^{2}+F_{x x}^{2} F^{2} \tilde{w}_{t}^{2}\right)+\left(F_{x}^{4} F_{t}^{2}+\tilde{w}_{t}^{2} F_{x}^{4}\right)\right. \\
& +\left(F_{t}^{2} F_{x}^{2} \tilde{w}_{x}^{2}+F^{2} \tilde{w}_{t}^{2} \tilde{w}_{x}^{2} F_{x}^{2}\right) \\
& \left.+\left(F^{4} F_{t}^{2}\left(\tilde{w}_{x x}^{2}+\tilde{w}_{x}^{4}\right)+\left(\tilde{w}_{t x x}^{2}+\tilde{w}_{x}^{2} \tilde{w}_{t x}^{2}\right) F^{4}\right)\right] \\
& =\left[\Gamma_{1}+\Gamma_{2}+\Gamma_{3}+\Gamma_{4}+\Gamma_{5}\right]+\left[\Delta_{1}+\Delta_{2}+\Delta_{3}+\Delta_{4}\right] \text {. }
\end{aligned}
$$


Then we bound each of these terms. Therefore, we have

$$
\begin{aligned}
& \int_{0}^{t} \int_{-\infty}^{+\infty} w_{4}(\tau) \Gamma_{1} d x d \tau \\
& \leq C \int_{0}^{t} \int_{-\infty}^{+\infty} w_{4}(\tau) F^{2} F_{t x x}^{2} d x d \tau \\
& \leq C \varepsilon^{2} \int_{0}^{t} w_{4}(\tau)\left\|F_{t x x}\right\|^{2} d \tau
\end{aligned}
$$

$$
\begin{aligned}
& \int_{0}^{t} \int_{-\infty}^{+\infty} w_{4}(\tau) \Gamma_{2} d x d \tau \\
& \leq C \int_{0}^{t} w_{4}(\tau)\left\|F_{t x}\right\|^{2}\left\|F_{x}\right\|^{2} d \tau+C \varepsilon^{2} \int_{0}^{t} w_{4}(\tau)\left\|F_{t x x}\right\|^{2} d \tau
\end{aligned}
$$

$$
\begin{gathered}
\int_{0}^{t} \int_{-\infty}^{+\infty} w_{4}(\tau) \Gamma_{3} d x d \tau \leq C \varepsilon^{2}, \\
\int_{0}^{t} \int_{-\infty}^{+\infty} w_{4}(\tau) \Gamma_{4} d x d \tau \leq C \delta^{2} \varepsilon^{2}, \\
\int_{0}^{t} \int_{-\infty}^{+\infty} w_{4}(\tau) \Gamma_{5} d x d \tau \leq C \delta^{2} \varepsilon^{2}, \\
\int_{0}^{t} \int_{-\infty}^{+\infty} w_{4}(\tau) \Delta_{1} d x d \tau \leq C\left(1+\delta^{2} \varepsilon^{2}\right), \\
\int_{0}^{t} \int_{-\infty}^{+\infty} w_{4}(\tau) \Delta_{2} d x d \tau \leq C
\end{gathered}
$$

$$
\begin{aligned}
& \int_{0}^{t} \int_{-\infty}^{+\infty} w_{4}(\tau) \Delta_{3} d x d \tau \\
& \leq C \delta^{2} \int_{0}^{t}\left(\left\|F_{x}\right\|^{2}+\left\|F_{x x}\right\|^{2}\right)\left(w_{3}(\tau)\left\|F_{t}\right\|^{2}\right) d \tau+C \delta^{2} \varepsilon^{2} \int_{0}^{t} w_{1}(\tau)\left\|F_{x}\right\|^{2} d \tau \\
& \leq C, \\
& \quad \int_{0}^{t} \int_{-\infty}^{+\infty} w_{4}(\tau) \Delta_{4} d x d \tau \\
& \quad \leq C \delta^{2} \int_{0}^{t}\left\|F_{t}\right\|^{2} w_{2}(\tau) d \tau+C \delta^{2} \int_{0}^{t} w_{1}(\tau)\|F\|^{2}\left\|F_{x}\right\|^{2} d \tau \\
& \leq C \delta^{2} .
\end{aligned}
$$


Thus, we see from (2.60)-(2.69) that

$$
\begin{aligned}
& w_{4}(t)\left\|F_{t x}(t)\right\|^{2}+\int_{0}^{t} w_{4}(\tau)\left\|F_{t x x}(\tau)\right\|^{2} d \tau \\
& \leq C+C \int_{0}^{t} w_{4}(\tau)\left\|F_{t x}\right\|^{2}\left\|F_{x}\right\|^{2} d \tau .
\end{aligned}
$$

Then the Gronwall inequality implies that

$$
w_{4}(t)\left\|F_{t x}(t)\right\|^{2}+\int_{0}^{t} w_{4}(\tau)\left\|F_{t x x}(\tau)\right\|^{2} d \tau \leq C .
$$

Equation (2.47) follows from (2.58) and (2.71).

COROLlary 2.11. The solution $F$ to (2.5), obtained in Theorem 2.8, satisfies

$$
\begin{gathered}
w_{3}(t)\left\|F_{x x}\right\|^{2} \leq C,\left\|F_{x x}\right\|_{L^{\infty}} \leq C\left(w_{3}(t) w_{4}(t)\right)^{-\frac{1}{4}}, \\
\left\|F_{x}\right\|_{L^{\infty}}^{2} \leq C w_{3}(t)^{-\frac{1}{2}} w_{2}(t)^{-\frac{1}{2}} .
\end{gathered}
$$

Proof. From (2.5), we see that

$$
\begin{aligned}
F_{x x}= & O(1)\left(F_{t}+\left(a-a_{1}\right) \tilde{w}_{t}+F \tilde{w}_{t}+F_{x}^{2}\right. \\
& \left.+F F_{x} \tilde{w}_{x}+\left(\tilde{w}_{x x}+\tilde{w}_{x}^{2}\right) F^{2}\right) .
\end{aligned}
$$

Taking the $L^{2}$-norm in (2.72), we have

$$
\begin{aligned}
w_{3}(t)\left\|F_{x x}\right\|^{2} \leq & C w_{3}(t)\left(\left\|F_{t}\right\|^{2}+\left\|\left(a-a_{1}\right) \tilde{w}_{t}\right\|^{2}+\left\|F \tilde{w}_{t}\right\|^{2}+\left\|F_{x}^{2}\right\|^{2}\right. \\
& \left.+\left\|F F_{x} \tilde{w}_{x}\right\|^{2}+\left\|\left(\tilde{w}_{x x}+\tilde{w}_{x}^{2}\right) F^{2}\right\|^{2}\right) \\
\leq & C\left(1+w_{3}(t)\left\|F_{x}^{2}\right\|^{2}\right) \\
\leq & C\left(1+w_{3}(t)\left\|F_{x}\right\|^{2}\left(\left\|F_{x}\right\|^{2}+\left\|F_{x x}\right\|^{2}\right)\right) \\
\leq & C+C w_{3}(t)\left\|F_{x}\right\|^{2}\left\|F_{x x}\right\|^{2}
\end{aligned}
$$

which implies

$$
w_{3}(t)\left\|F_{x x}\right\|^{2} \leq C .
$$

Then

$$
\left\|F_{x}\right\|_{L^{\infty}}^{2} \leq C w_{3}(t)^{-\frac{1}{2}} w_{2}(t)^{-\frac{1}{2}} .
$$

Last, if we take the $L^{\infty}$-norm in (2.72), we obtain

$$
\begin{gathered}
\left\|F_{x x}\right\|_{L^{\infty}} \leq C\left(\left\|F_{t}\right\|_{L^{\infty}}+\left\|\left(a-a_{1}\right) \tilde{w}_{t}\right\|_{L^{\infty}}+\left\|F_{x}\right\|_{L^{\infty}}^{2}\right. \\
\left.+\left\|F F_{x} \tilde{w}_{x}\right\|_{L^{\infty}}+\left\|F^{2}\left(\tilde{w}_{x x}+\tilde{w}_{x}^{2}\right)\right\|_{L^{\infty}}\right) \\
\leq C\left(w_{3}(t) w_{4}(t)\right)^{-\frac{1}{4}} \cdot \quad
\end{gathered}
$$


Then Theorem 2.2 follows from Theorem 2.8, Lemmas 2.9-2.10, and Corollary 2.11 .

Now, from $F$ it is easy to obtain the solution $\phi$ of (2.4) and from $\phi$ the unique smooth solution $w$ of (2.2). By defining $\tilde{v}=a^{-1}(x) w$ and $\tilde{u}=-\left(w^{-\gamma}\right)_{x}$, we obtain the solution of (1.4). Theorem 1.1 then follows from Theorem 2.2 and the decay estimates follow from the interpolation inequality and (2.35).

2.2. Case 2: $\boldsymbol{w}_{-}=\boldsymbol{w}_{+}=$const $=\overline{\boldsymbol{w}}$. Observe that $w=\bar{w}$ is a stationary solution to (2.2). We will prove Theorem 1.2 by solving the Cauchy problem (2.2) near $\bar{w}$.

Let us denote by $\tilde{\phi}=w-\bar{w}$, then $\tilde{\phi}$

$$
\left\{\begin{array}{l}
\tilde{\phi}_{t}-b a(x) \tilde{\phi}_{x x}+a(x)\left(f_{1}(\tilde{\phi}) \tilde{\phi}^{2}\right)_{x x}=0 \\
\tilde{\phi}(x, 0)=\tilde{\phi}_{0}(x)=w_{0}(x)-\bar{w}
\end{array}\right.
$$

where

$$
b=\gamma \bar{w}^{-(\gamma+1)}, f_{1} \tilde{\phi}^{2}=(\bar{w}+\tilde{\phi})^{-\gamma}-\bar{w}^{-\gamma}-b \tilde{\phi} .
$$

Then we have the following.

TheOREM 2.12. Suppose $\tilde{\phi}_{0}(x)$ and $s(x)=s_{0}(x)$ are $C^{2}$ functions and $\tilde{\phi}_{0} \in$ $H^{2}(R)$. There exists $\varepsilon_{0}>0$ such that if $0<\varepsilon<\varepsilon_{0}$ and $\left\|\tilde{\phi}_{0}\right\|_{H^{2}} \leq \varepsilon$, then (2.73) has a unique global smooth solution $\tilde{\phi}(x, t)$ satisfying

$$
\|\tilde{\phi}(\cdot, t)\|_{H^{2}}^{2}+\int_{0}^{t}\left\|\tilde{\phi}_{x}(\cdot, \tau)\right\|_{H^{2}}^{2} d \tau \leq C \varepsilon^{2}
$$

and

$$
\lim _{t \rightarrow \infty}\|\tilde{\phi}(\cdot, t)\|_{L^{\infty}}=0 .
$$

Furthermore, if $\tilde{\phi}_{0} \in L^{1}(R)$, then

$$
\sum_{j=0}^{2} w_{j+1}(t)\left\|\partial_{x}^{j} \tilde{\phi}(\cdot, t)\right\|^{2}+\int_{0}^{t} \sum_{j=1}^{3} w_{j}(\tau)\left\|\partial_{x}^{j} \tilde{\phi}(\cdot, \tau)\right\|^{2} d \tau \leq C .
$$

Proof. Since the local result for (2.73) is classical, to prove the first part of Theorem 2.12, it is sufficient to derive the uniform estimate (2.74) under the a priori assumption $\|\tilde{\phi}\|_{H^{2}} \leq \delta_{0}$ for $\delta_{0}$ suitably small.

Multiply $(2.73)_{1}$ by $a^{-1} \tilde{\phi}$, integrate it over $(-\infty,+\infty) \times[0, t]$, and one then has

$$
\begin{aligned}
& \|\tilde{\phi}(\cdot, t)\|^{2}+\int_{0}^{t}\left\|\tilde{\phi}_{x}(\cdot, \tau)\right\|^{2} d \tau \\
& \leq C \varepsilon_{0}^{2}+C\left|\int_{0}^{t} \int_{-\infty}^{+\infty}\left(f_{1}(\tilde{\phi}) \tilde{\phi}^{2}\right)_{x} \tilde{\phi}_{x} d x d \tau\right| \\
& \leq C \varepsilon_{0}^{2}+C \delta_{0} \int_{0}^{t}\left\|\tilde{\phi}_{x}\right\|^{2} d \tau,
\end{aligned}
$$

which implies

$$
\|\tilde{\phi}(\cdot, t)\|^{2}+\int_{0}^{t}\left\|\tilde{\phi}_{x}(\cdot, \tau)\right\|^{2} d \tau \leq C \varepsilon_{0}^{2} .
$$


Next, multiplying $(2.73)_{1}$ by $\tilde{\phi}_{x x}$ and integrating over $(-\infty,+\infty) \times[0, t]$, one has

$$
\begin{aligned}
& \left\|\tilde{\phi}_{x}(\cdot, t)\right\|^{2}+\int_{0}^{t}\left\|\tilde{\phi}_{x x}(\cdot, \tau)\right\|^{2} d \tau \\
& \leq C \varepsilon_{0}^{2}+C\left|\int_{0}^{t} \int_{-\infty}^{+\infty}\left(f_{1}(\tilde{\phi}) \tilde{\phi}^{2}\right)_{x x} \tilde{\phi}_{x x} d x d \tau\right| \\
& \leq C \varepsilon_{0}^{2}+\frac{1}{2} \int_{0}^{t}\left\|\tilde{\phi}_{x x}(\cdot, \tau)\right\|^{2} d \tau+C \int_{0}^{t} \int_{-\infty}^{+\infty}\left(f_{1}(\tilde{\phi}) \tilde{\phi}^{2}\right)_{x x}^{2} d x d \tau \\
& \leq C \varepsilon_{0}^{2}+\left(\frac{1}{2}+C \delta_{0}\right) \int_{0}^{t}\left\|\tilde{\phi}_{x x}(\cdot, \tau)\right\|^{2} d \tau .
\end{aligned}
$$

Thus we get

$$
\left\|\tilde{\phi}_{x}(\cdot, t)\right\|^{2}+\int_{0}^{t}\left\|\tilde{\phi}_{x x}(\cdot, \tau)\right\|^{2} d \tau \leq C \varepsilon_{0}^{2} .
$$

Finally, by differentiating $(2.73)_{1}$ in $x$ and by repeating the previous procedure, one can derive

$$
\left\|\tilde{\phi}_{x x}(\cdot, t)\right\|^{2}+\int_{0}^{t}\left\|\tilde{\phi}_{x x x}(\cdot, \tau)\right\|^{2} d \tau \leq C \varepsilon_{0}^{2} .
$$

The estimate (2.74) follows from (2.75)-(2.77) and then (2.73) has a unique smooth solution $\tilde{\phi}$ such that

$$
\lim _{t \rightarrow \infty}\|\tilde{\phi}(\cdot, t)\|_{L^{\infty}}=0 .
$$

We proceed now to prove the second part of Theorem 2.12. In this framework, we can develop a theory similar to what we did in the previous sections. Actually, it is less complicated since $\bar{w}$ is a constant.

Observe that if $\tilde{\phi}_{0}(x) \in L^{1}$, we can use the same argument used in Lemma 2.3 to prove

$$
\|\tilde{\phi}(\cdot, t)\|_{L^{1}} \leq\left\|\tilde{\phi}_{0}\right\|_{L^{1}} .
$$

Then we can employ the same argument used in subsection 2.1 to complete the proof of the decay estimates. We perform here just the first two orders estimates.

For the first order estimates, we multiply $(2.73)_{1}$ by $w_{1}(t) a^{-1} \tilde{\phi}$ then integrate it by parts over $(-\infty,+\infty) \times[0, t]$. We have

$$
\begin{aligned}
& w_{1}(t)\|\tilde{\phi}(\cdot, t)\|^{2}+\int_{0}^{t} w_{1}(\tau)\left\|\tilde{\phi}_{x}(\cdot, \tau)\right\|^{2} d \tau \\
& \leq C+C \int_{0}^{t}(1+\tau)^{-1} w_{1}(\tau)\|\tilde{\phi}(\cdot, \tau)\|_{L^{\infty}}\|\tilde{\phi}(\cdot, \tau)\|_{L^{1}} d \tau \\
& \quad+C \delta_{0} \int_{0}^{t} w_{1}(\tau)\left\|\tilde{\phi}_{x}(\cdot, \tau)\right\|^{2} d \tau,
\end{aligned}
$$

where

$$
\begin{aligned}
& \int_{0}^{t}(1+\tau)^{-1} w_{1}(\tau)\|\tilde{\phi}(\cdot, \tau)\|_{L^{\infty}}\|\tilde{\phi}(\cdot, \tau)\|_{L^{1}} d \tau \\
& \leq C\left(\varepsilon_{1}\right) \int_{0}^{t} w_{1}(\tau)(1+t)^{-\frac{3}{2}} d \tau+\varepsilon_{1} \int_{0}^{t} w_{1}(\tau)\left\|\tilde{\phi}_{x}(\cdot, \tau)\right\|^{2} d \tau
\end{aligned}
$$


By choosing $\varepsilon_{1}$ small, we see from (2.79)-(2.80)

$$
w_{1}(t)\|\tilde{\phi}(\cdot, t)\|^{2}+\int_{0}^{t} w_{1}(\tau)\left\|\tilde{\phi}_{x}(\cdot, \tau)\right\|^{2} d \tau \leq C .
$$

For the second order estimates, we multiply $(2.73)_{1}$ by $w_{2}(t) \tilde{\phi}_{x x}$, integrate it by parts over $(-\infty,+\infty) \times[0, t]$, then

$$
\begin{aligned}
& w_{2}(t)\left\|\tilde{\phi}_{x}(\cdot, t)\right\|^{2}+\int_{0}^{t} w_{2}(\tau)\left\|\tilde{\phi}_{x x}(\cdot, \tau)\right\|^{2} d \tau \\
& \leq C+\frac{1}{2} \int_{0}^{t} w_{2}(\tau)\left\|\tilde{\phi}_{x x}(\cdot, \tau)\right\|^{2} d \tau \\
& \quad+C \int_{0}^{t} \int_{-\infty}^{+\infty} w_{2}(\tau)\left(f_{1}(\tilde{\phi}) \tilde{\phi}^{2}\right)_{x x}^{2} d x d \tau,
\end{aligned}
$$

where

$$
\begin{aligned}
& \int_{0}^{t} \int_{-\infty}^{+\infty} w_{2}(\tau)\left(f_{1}(\tilde{\phi}) \tilde{\phi}^{2}\right)_{x x}^{2} d x d \tau \\
& \leq C \delta_{0} \int_{0}^{t} w_{2}(\tau)\left\|\tilde{\phi}_{x x}(\cdot, \tau)\right\|^{2} d \tau \\
& \quad+C \int_{0}^{t} w_{2}(\tau)\left\|\tilde{\phi}_{x}(\tau)\right\|^{2}\left(\left\|\tilde{\phi}_{x}\right\|^{2}+\left\|\tilde{\phi}_{x x}\right\|^{2}\right) d \tau \\
& \leq C \delta_{0} \int_{0}^{t} w_{2}(\tau)\left\|\tilde{\phi}_{x x}(\cdot, \tau)\right\|^{2} d \tau+C \int_{0}^{t} w_{2}(\tau)\left\|\tilde{\phi}_{x}(\tau)\right\|^{4} d \tau .
\end{aligned}
$$

We conclude from (2.82)-(2.83) that

$$
\begin{aligned}
& w_{2}(t)\left\|\tilde{\phi}_{x}(\cdot, t)\right\|^{2}+\int_{0}^{t} w_{2}(\tau)\left\|\tilde{\phi}_{x x}(\cdot, \tau)\right\|^{2} d \tau \\
& \leq C+C \int_{0}^{t} w_{2}(\tau)\left\|\tilde{\phi}_{x}(\tau)\right\|^{4} d \tau
\end{aligned}
$$

which, together with the help of Gronwall inequality, implies that

$$
w_{2}(t)\left\|\tilde{\phi}_{x}(\cdot, t)\right\|^{2}+\int_{0}^{t} w_{2}(\tau)\left\|\tilde{\phi}_{x x}(\cdot, \tau)\right\|^{2} d \tau \leq C .
$$

In the following, we denote by $v_{2}(x, t)=a^{-1} w(x, t)$ the solution to (2.1) obtained in Theorem 1.2, and $u_{2}(x, t)=-p\left(v_{2}, s\right)_{x}$. Theorem 1.2 then follows from Theorem 2.12 .

3. Convergence to similarity solutions. In this section, we will study (1.2)(1.3) for Case 1, namely, we assume that $s_{-}=s_{+}=\bar{s}$. We shall prove Theorem 1.3 by comparing the solutions of (1.2)-(1.3) with those of (1.4) obtained in Theorem 2.2. Since the result for $s(x, t)$ is clear, in the following part we only deal with $(v, u)(x, t)$.

Let $(\tilde{v}, \tilde{u}, s(x))$ be the solution of (1.4) with the initial data $\left(\tilde{v}_{0}(x), s_{0}(x)\right)$. As pointed in introduction, we will only prove Theorem 1.3 for the case where $u_{-}=$ 
$u_{+}=0$ and thus (1.14) turns into

$$
\int_{-\infty}^{+\infty}\left(v_{0}(x)-\tilde{v}_{0}(x)\right) d x=0 .
$$

Let us denote

$$
v_{e}=v-\tilde{v}, u_{e}=u-\tilde{u},
$$

then it follows from (1.2) and (1.4) that

$$
\left\{\begin{array}{l}
v_{e t}-u_{e x}=0 \\
u_{e t}+\left[p\left(\tilde{v}+v_{e}, s\right)-p(\tilde{v}, s)\right]_{x}=-u_{e}+p(\tilde{v}, s)_{x t} .
\end{array}\right.
$$

As usual let us consider

$$
y=\int_{-\infty}^{x} v_{e}(\xi) d \xi
$$

which satisfies the following nonlinear wave equation:

$$
\left\{\begin{array}{l}
y_{t t}+\left[p\left(y_{x}+\tilde{v}, s\right)-p(\tilde{v}, s)\right]_{x}+y_{t}=p(\tilde{v}, s)_{x t}, \\
y(x, 0)=y_{0}(x)=\int_{-\infty}^{x}\left(v_{0}-\tilde{v}_{0}\right)(\xi) d \xi \\
y_{t}(x, 0)=y_{1}(x)=u_{0}(x)-\tilde{u}(x, 0)
\end{array}\right.
$$

since $y_{x}=v_{e}$ and $y_{t}=u_{e}$. Therefore

$$
\left\{\begin{array}{l}
y_{t t}+\left(p_{v}(\tilde{v}, s) y_{x}\right)_{x}+y_{t}=p(\tilde{v}, s)_{x t}-\left(F_{1}\left(\tilde{v}, y_{x}, s\right) y_{x}^{2}\right)_{x}, \\
y(x, 0)=y_{0}(x)=\int_{-\infty}^{x}\left(v_{0}-\tilde{v}_{0}\right)(\xi) d \xi \\
y_{t}(x, 0)=y_{1}(x)=u_{0}(x)-\tilde{u}(x, 0),
\end{array}\right.
$$

where

$$
p\left(y_{x}+\tilde{v}, s\right)-p(\tilde{v}, s)=p_{v}(\tilde{v}, s) y_{x}+F_{1}\left(\tilde{v}, y_{x}, s\right) y_{x}^{2} .
$$

The main result of this section is the following.

TheOrem 3.1. There exists $\delta_{0}>0$ such that if $0<\delta<\delta_{0}$ and

$$
\left\|y_{0}\right\|_{H^{3}}+\left\|y_{1}\right\|_{H^{2}}+\left|v_{+}-v_{-}\right| \leq \delta,
$$

then (3.5) has a unique smooth solution $y \in H^{3}$ and $y_{t} \in H^{2}$ satisfying

$$
\|y(t)\|_{H^{3}}^{2}+\left\|y_{t}(t)\right\|_{H^{2}}^{2}+\int_{0}^{t}\left\|\left(y_{x}, y_{t}\right)(\tau)\right\|_{H^{2}} d \tau \leq C \delta^{2} .
$$

Moreover,

$$
(1+t)\left\|y_{x}(\cdot, t)\right\|^{2}+(1+t)^{2}\left\|y_{t}(\cdot, t)\right\|^{2} \leq C
$$


and

$$
\left\|y_{x}(\cdot, t)\right\|_{L^{\infty}} \leq C(1+t)^{-\frac{3}{4}},\left\|y_{t}(\cdot, t)\right\|_{L^{\infty}} \leq C(1+t)^{-\frac{5}{4}} .
$$

Then Theorem 1.3 follows from Theorem 2.2 and Theorem 3.1.

We now prove Theorem 3.1. First of all, we have the following.

THEOREM 3.2. There exists $\delta_{0}>0$ such that if $0<\delta<\delta_{0}$ and

$$
\left\|y_{0}\right\|_{H^{3}}+\left\|y_{1}\right\|_{H^{2}}+\left|v_{+}-v_{-}\right| \leq \delta,
$$

then (3.5) has a unique global smooth solution $y \in H^{3}$ and $y_{t} \in H^{2}$ satisfying

$$
\|y(t)\|_{H^{3}}^{2}+\left\|y_{t}(t)\right\|_{H^{2}}^{2}+\int_{0}^{t}\left\|\left(y_{x}, y_{t}\right)(\tau)\right\|_{H^{2}} d \tau \leq C \delta^{2} .
$$

Proof. It is sufficient to prove the uniform estimates (3.8) under the following a priori assumption:

$$
\|y(t)\|_{H^{3}}^{2}+\left\|y_{t}\right\|_{H^{2}}^{2} \leq \varepsilon
$$

for $\varepsilon>0$ suitably small.

Multiplying $(3.5)_{1}$ by $y+2 y_{t}$, we have

$$
\begin{aligned}
& {\left[y_{t}^{2}-p_{v}(\tilde{v}, s) y_{x}^{2}+\frac{1}{2} y^{2}+y y_{t}\right]_{t}+y_{t}^{2}-p_{v}(\tilde{v}, s) y_{x}^{2}} \\
& =p_{v v}(\tilde{v}, s) \tilde{v}_{t}\left(y_{x}^{2}-y_{x}-2 y_{x t}\right)+\left(F_{1} y_{x}^{2}-p(\tilde{v}, s)_{t}\right)\left(y_{x}+2 y_{t x}\right)+\{\cdots\}_{x},
\end{aligned}
$$

where $\{\cdots\}_{x}$ denote the terms which disappear after integration with respect to $x$. Integrating $(3.9)$ over $[0, t] \times(-\infty,+\infty)$, we get

$$
\begin{aligned}
& \left\|\left(y, y_{t}, y_{x}\right)(t)\right\|^{2}+\int_{0}^{t}\left\|\left(y_{t}, y_{x}\right)(\tau)\right\|^{2} d \tau \\
& \leq C \delta^{2}+C \mid \int_{0}^{t} \int_{-\infty}^{+\infty} p_{v v}(\tilde{v}, s) \tilde{v}_{t} y_{x}^{2} \\
& \quad-p(\tilde{v}, s)_{t}\left(y_{x}+2 y_{x t}\right)+F_{1} y_{x}^{2}\left(y_{x}+2 y_{t x}\right) d x d \tau \mid .
\end{aligned}
$$

Due to the smallness of $\varepsilon$, we can reduce (3.10) into

$$
\begin{aligned}
& \left\|\left(y, y_{t}, y_{x}\right)(t)\right\|^{2}+\int_{0}^{t}\left\|\left(y_{t}, y_{x}\right)(\tau)\right\|^{2} d \tau \\
& \leq C \delta^{2}+C\left|\int_{0}^{t} \int_{-\infty}^{+\infty} p(\tilde{v}, s)_{t}\left(y_{x}+2 y_{x t}\right) d x d \tau\right|
\end{aligned}
$$

while

$$
\begin{aligned}
& \left|\int_{0}^{t} \int_{-\infty}^{+\infty} p(\tilde{v}, s)_{t}\left(y_{x}+2 y_{x t}\right) d x d \tau\right| \\
& \leq C\left(\varepsilon_{1}\right) \int_{0}^{t} \int_{-\infty}^{+\infty}\left(p(\tilde{v}, s)_{t}^{2}+p(\tilde{v}, s)_{t x}^{2}\right) d x d \tau+\varepsilon_{1} \int_{0}^{t} \int_{-\infty}^{+\infty}\left(y_{x}^{2}+y_{t}^{2}\right) d x d \tau
\end{aligned}
$$


and

$$
\int_{0}^{t} \int_{-\infty}^{+\infty} p(\tilde{v}, s)_{t}^{2}+p(\tilde{v}, s)_{t x}^{2} d x d \tau \leq C \delta^{2}
$$

Now, by taking $\varepsilon_{1}$ small, it reads from (3.11)-(3.13) that

$$
\left\|\left(y, y_{t}, y_{x}\right)(t)\right\|^{2}+\int_{0}^{t}\left\|\left(y_{x}, y_{t}\right)(\tau)\right\|^{2} d \tau \leq C \delta^{2} .
$$

We now differentiate (3.5) in $x$ and then

$$
y_{t t x}+\left(p_{v}(\tilde{v}, s) y_{x}\right)_{x x}+y_{t x}=p(\tilde{v}, s)_{x t x}-\left(F_{1}\left(\tilde{v}, y_{x}, s\right) y_{x}^{2}\right)_{x x}
$$

If we multiply (3.15) by $y_{x}+2 y_{t x}$ and integrate the resulting equation over $[0,1] \times[0, t]$, by using (3.14) we get

$$
\begin{aligned}
& \left\|\left(y_{x}, y_{t x}, y_{x x}\right)(t)\right\|^{2}+\int_{0}^{t}\left\|\left(y_{t x}, y_{x x}\right)(\tau)\right\|^{2} d \tau \\
& \leq C \delta^{2}+C \int_{0}^{t} \int_{-\infty}^{+\infty}\left(F_{1} y_{x}^{2}\right)_{x}^{2} d x d \tau \\
& \quad+C \int_{0}^{t} \int_{-\infty}^{+\infty}\left[O(1) y_{x} y_{x x}^{2}\right]_{t} d x d \tau \\
& +C \delta \int_{0}^{t} \int_{-\infty}^{+\infty}\left(y_{x}^{2}+y_{t x}^{2}+y_{x x}^{2}\right) d x d \tau,
\end{aligned}
$$

which implies

$$
\left\|\left(y_{x}, y_{t x}, y_{x x}\right)(t)\right\|^{2}+\int_{0}^{t}\left\|\left(y_{t x}, y_{x x}\right)(\tau)\right\|^{2} d \tau \leq C \delta^{2} .
$$

Repeating the above procedure, we can easily obtain the third order estimates and complete the proof of this theorem.

With the help of Theorem 3.2, it is easy to obtain the following convergence results by using an argument similar to the proof of Theorem 2.8.

TheOREM 3.3. The solution y to (3.5) in the Theorem 3.2 satisfies

$$
\lim _{t \rightarrow \infty}\left(\|y(\cdot, t)\|_{L^{\infty}}+\left\|\left(y_{t}, y_{x}\right)(\cdot, t)\right\|_{H^{1}}\right)=0 .
$$

We investigate now the problem of the decay rate. We will follow the approach introduced by [19] concerning the isentropic case. However, since the entropy $s(x)$ is not constant here, some modifications are necessary.

LEMma 3.4. Under the previous hypotheses, it follows that

$$
(1+t)\left\|\left(y_{x}, y_{t}\right)(t)\right\|^{2}+\int_{0}^{t}(1+\tau)\left\|y_{t}(\tau)\right\|^{2} d \tau \leq C \delta^{2} .
$$

Proof. First, we notice that $(3.5)_{1}$ is equivalent to

$$
y_{t t}+y_{t}+\left[p\left(\tilde{v}+y_{x}, s\right)-p(\tilde{v}, s)\right]_{x}=p(\tilde{v}, s)_{x t} .
$$


Multiplying (3.16) by $(1+t) y_{t}$, after some calculations we get

$$
\begin{aligned}
& {\left[(1+t)\left(\frac{1}{2} y_{t}^{2}+q\right)\right]_{t}+(1+t) y_{t}^{2}-q} \\
& -\int_{0}^{y_{x}}\left[p_{v}(\tilde{v}+\xi, s)-p_{v}(\tilde{v}, s)\right] d \xi+\tilde{v}_{t}(1+t) y_{x}^{2}-\frac{1}{2} y_{t}^{2} \\
& =(1+t) y_{t} p(\tilde{v}, s)_{x t}+\{\cdots\}_{x} .
\end{aligned}
$$

Integrating (3.17) over $[0, t] \times(-\infty,+\infty)$, with the help of $(3.8)$, we have

$$
\begin{aligned}
& (1+t)\left\|\left(y_{x}, y_{t}\right)(t)\right\|^{2}+\int_{0}^{t}(1+\tau)\left\|y_{t}(\tau)\right\|^{2} d \tau \\
& \leq C \delta^{2}+\frac{1}{2} \int_{0}^{t}(1+\tau)\left\|y_{t}(\tau)\right\|^{2} d \tau
\end{aligned}
$$

which implies

$$
(1+t)\left\|\left(y_{t}, y_{x}\right)(t)\right\|^{2}+\int_{0}^{t}(1+\tau)\left\|y_{t}(\tau)\right\|^{2} d \tau \leq C \delta^{2} .
$$

Here we have used the following properties:

$$
\begin{aligned}
& q=-\int_{0}^{y_{x}}[p(\tilde{v}+\xi, s)-p(\tilde{v}, s)] d \xi=O(1) y_{x}^{2} \\
& \int_{0}^{y_{x}}\left[p_{v}(\tilde{v}+\xi, s)-p_{v}(\tilde{v}, s)\right] d \xi=O(1) y_{x}^{2} \\
& \tilde{v}_{t} \leq O(1)\left(F_{t}+\tilde{w}_{t}\right) \leq O(1)(1+t)^{-1} .
\end{aligned}
$$

Lemma 3.5. Under the previous hypotheses, we have

$$
(1+t)^{2}\left\|\left(y_{t}, y_{t t}, y_{t x}\right)(t)\right\|^{2}+\int_{0}^{t}(1+\tau)^{2}\left\|\left(y_{t t}, y_{t x}\right)(\tau)\right\|^{2} d \tau \leq C \delta^{2} .
$$

Proof. Differentiating $(3.5)_{1}$ in $t$, we have

$$
y_{t t t}+\left(p_{v}(\tilde{v}, s) y_{x}\right)_{x t}+y_{t t}=p(\tilde{v}, s)_{x t t}-\left(F_{1} y_{x}^{2}\right)_{x t} .
$$

Let us multiply $(3.19)$ by $(1+t) y_{t}$ and $(1+t) y_{t t}$, respectively, then we deduce

$$
\begin{aligned}
& {\left[(1+t)\left(y_{t} y_{t t}+\frac{1}{2} y_{t}^{2}\right)\right]_{t}-p_{v}(\tilde{v}, s)(1+t) y_{t x}^{2}-(1+t) y_{t t}^{2}-\frac{1}{2} y_{t}^{2}-y_{t} y_{t t}} \\
& =p_{v v} \tilde{v}_{t}(1+t) y_{x} y_{t x}+(1+t) y_{t}\left(p(\tilde{v}, s)_{x t t}-\left(F_{1} y_{x}^{2}\right)_{x t}\right)+\{\cdots\}_{x}, \\
& \quad\left[\frac{1}{2}(1+t)\left(y_{t t}^{2}-p_{v} y_{t x}^{2}\right)\right]_{t}+(1+t) y_{t t}^{2} \\
& \quad-\frac{1}{2} y_{t t}^{2}+\frac{1}{2} p_{v} y_{t x}^{2}+\frac{1}{2}(1+t) p_{v v} \tilde{v}_{t} y_{t x}^{2}+(1+t) y_{t t}\left(y_{x} p_{v v} \tilde{v}_{t}\right)_{x} \\
& =(1+t) y_{t t}\left(p(\tilde{v}, s)_{x t t}-\left(F_{1} y_{x}^{2}\right)_{x t}\right)+\{\cdots\}_{x} .
\end{aligned}
$$


By using Theorem 3.2 and Lemma 3.4 and by integrating $8 \times(3.21)+(3.20)$ one has

$$
\begin{aligned}
& (1+t)\left\|\left(y_{t}, y_{t t}, y_{t x}\right)\right\|^{2}+\int_{0}^{t}(1+\tau)\left\|\left(y_{t t}, y_{t x}\right)(\tau)\right\|^{2} d \tau \\
& \leq C\left(\delta^{2}+\int_{0}^{t} \int_{-\infty}^{+\infty}(1+\tau) p(\tilde{v}, s)_{x t t}^{2} d x d \tau\right. \\
& \left.+\left|\int_{0}^{t} \int_{-\infty}^{+\infty}(1+\tau)\left(y_{t x}+y_{t t x}\right)\left(F_{1} y_{x}^{2}\right)_{t} d x d \tau\right|\right) \\
& \leq C \delta^{2}+C\left|\int_{0}^{t} \int_{-\infty}^{+\infty}(1+\tau)\left(y_{t x}+y_{t t x}\right)\left(F_{1} y_{x}^{2}\right)_{t} d x d \tau\right| \text {. }
\end{aligned}
$$

Moreover, one has

$$
\begin{aligned}
& \left|\int_{0}^{t} \int_{-\infty}^{+\infty}(1+\tau) y_{t x}\left(F_{1} y_{x}^{2}\right)_{t} d x d \tau\right| \\
& \leq C \int_{0}^{t} \int_{-\infty}^{+\infty}(1+\tau)\left(\left|y_{x}\right| y_{t x}^{2}+\left|\tilde{v}_{t} y_{x}^{2} y_{t x}\right|\right) d x d \tau \\
& \leq C \delta^{2}+C \delta \int_{0}^{t} \int_{-\infty}^{+\infty}(1+\tau) y_{t x}^{2} d x d \tau
\end{aligned}
$$

and

$$
\begin{aligned}
& \left|\int_{0}^{t} \int_{-\infty}^{+\infty}(1+\tau) y_{t t x}\left(F_{1} y_{x}^{2}\right)_{t} d x d \tau\right| \\
& \leq C \delta^{2}+C \delta\left((1+t)\left\|y_{t x}(t)\right\|^{2}+\int_{0}^{t}(1+\tau)\left\|y_{t x}\right\|^{2} d \tau\right) .
\end{aligned}
$$

In view of the smallness of $\delta$, from (3.22)-(3.24) we have

$$
(1+t)\left\|\left(y_{t}, y_{t t}, y_{t x}\right)(t)\right\|^{2}+\int_{0}^{t}(1+\tau)\left\|\left(y_{t t}, y_{t x}\right)(\tau)\right\|^{2} d \tau \leq C \delta^{2} .
$$

Now we multiply (3.19) by $(1+t)^{2} y_{t}$ and $(1+t)^{2} y_{t t}$ and repeat the previous calculations to conclude Lemma 3.5 .

Lemma 3.6. The solution y to (3.5) in Theorem 3.2 satisfies

$$
(1+t)^{2}\left\|\left(V_{t}, V_{x}\right)(t)\right\|^{2}+\int_{0}^{t}(1+\tau)\left\|\left(V_{t}, V_{x}\right)(\tau)\right\|^{2} d \tau \leq C \delta^{2}
$$

where $V=p_{v}(\tilde{v}, s) y_{x}$.

Proof. The estimate for $V_{t}$ can be obtained from Lemma 3.5 and the following relation:

$$
V_{t}=p_{v}(\tilde{v}, s) y_{t x}+p_{v v}(\tilde{v}, s) \tilde{v}_{t} y_{x}
$$

It is easy to see that

$$
V_{x}=-\left(y_{t t}+y_{t}+p(\tilde{v}, s)_{x t}+\left(F_{2} V^{2}\right)_{x}\right)
$$


where $F_{2} V^{2}=F_{1} y_{x}^{2}$. Then we calculate the decay rate for $V_{x}$ by using (3.26). First of all, it is easy to see by taking the $L^{2}$-norm in (3.26) that

$$
(1+t)\left\|V_{x}(t)\right\|^{2} \leq C \delta^{2}
$$

and

$$
\begin{aligned}
& (1+t)^{2}\left\|V_{x}\right\|^{2} \\
& \leq C(1+t)^{2}\left(\left\|y_{t t}\right\|^{2}+\left\|y_{t}\right\|^{2}+\left\|\left(F_{2} V^{2}\right)_{x}\right\|^{2}\right) \\
& \leq C \delta^{2}+\frac{1}{2}(1+t)^{2}\left\|V_{x}\right\|^{2}+C(1+t)\left(\|V\|^{2}+\left\|V_{x}\right\|^{2}\right) \\
& \leq C \delta^{2}+\frac{1}{2}(1+t)^{2}\left\|V_{x}\right\|^{2}
\end{aligned}
$$

thus

$$
(1+t)^{2}\left\|V_{x}\right\|^{2} \leq C \delta^{2} .
$$

Then, multiplying (3.26) by $(1+t) V_{x}$ and integrating it, one has

$$
\begin{aligned}
& \int_{0}^{t}(1+\tau)\left\|V_{x}(\tau)\right\|^{2} d \tau \\
& \leq C \int_{0}^{t} \int_{-\infty}^{+\infty}(1+\tau)\left(y_{t t}^{2}+y_{t}^{2}+p(\tilde{v}, s)_{x t}^{2}+\left(F_{2} V^{2}\right)_{x}^{2}\right) d x d \tau \\
& \leq C \delta^{2}+C \int_{0}^{t} \int_{-\infty}^{+\infty}\left(F_{2} V^{2}\right)_{x}^{2}(1+\tau) d x d \tau \\
& \leq C \delta^{2}+C \int_{0}^{t} \int_{-\infty}^{+\infty}(1+\tau) V^{4} d x d \tau \\
& \leq C \delta^{2}+C \int_{0}^{t}\left(\left\|y_{x}\right\|^{2}+\left\|y_{x x}\right\|^{2}\right)(1+\tau)\left\|y_{x}\right\|^{2} d \tau \\
& \leq C \delta^{2} .
\end{aligned}
$$

The following result easily holds by repeating the previous arguments on the equation (3.19) differentiated with respect to $x$.

Lemma 3.7. The solution y to (3.5) in Theorem 3.2 satisfies

$$
(1+t)^{2}\left\|\left(y_{t t x}, y_{t x x}\right)(t)\right\|^{2}+\int_{0}^{t}(1+\tau)^{2}\left\|\left(y_{t t x}, y_{t x x}\right)(\tau)\right\|^{2} d \tau \leq C \delta^{2} .
$$

Now we can prove the desired estimates on $y_{t x}$.

LEMMA 3.8. Under the previous hypotheses, one has

$$
(1+t)^{3}\left\|\left(y_{t t}, y_{t x}\right)(t)\right\|^{2}+\int_{0}^{t}(1+\tau)^{3}\left\|y_{t t}(\tau)\right\|^{2} d \tau \leq C \delta^{2} .
$$

Proof. Multiply (3.19) by $(1+t)^{3} y_{t t}$, then we obtain

$$
\begin{aligned}
& (1+t)^{3}\left\|\left(y_{t t}, y_{t x}\right)(t)\right\|^{2}+\int_{0}^{t}(1+\tau)^{3}\left\|y_{t t}(\tau)\right\|^{2} d \tau \\
& \leq C \delta^{2}+C\left|\int_{0}^{t} \int_{-\infty}^{+\infty}(1+\tau)^{3} y_{t t x}\left(F_{1} y_{x}^{2}\right)_{t} d x d \tau\right| .
\end{aligned}
$$


We have that

$$
\begin{aligned}
& \left|\int_{0}^{t} \int_{-\infty}^{+\infty}(1+\tau)^{3} y_{t t x}\left(F_{1} y_{x}^{2}\right)_{t} d x d \tau\right| \\
& \leq C\left|\int_{0}^{t} \int_{-\infty}^{+\infty}\left[O(1)(1+\tau)^{3} y_{t x}^{2} y_{x}\right]_{t} d x d \tau\right| \\
& +C \mid \int_{0}^{t} \int_{-\infty}^{+\infty}\left((1+t)^{2} y_{t x}^{2}+(1+\tau)^{3} y_{t x}^{3} d x d \tau \mid\right. \\
& +C\left|\int_{0}^{t} \int_{-\infty}^{+\infty}(1+\tau)^{3} y_{t t}\left(F_{3} \tilde{v}_{t} V^{2}\right)_{x} d x d \tau\right| \\
& \leq C\left(\alpha_{1}\right) \delta^{2}+C \delta(1+t)^{3}\left\|y_{t x}(t)\right\|^{2}+\alpha_{1} \int_{0}^{t}(1+\tau)^{3}\left\|y_{t t}\right\|^{2} d \tau
\end{aligned}
$$

where $F_{3} V^{2}=F_{1 v} y_{x}^{2}$.

By choosing $\alpha_{1}$ suitable small, we conclude from (3.30)-(3.31) that

$$
(1+t)^{3}\left\|\left(y_{t t}, y_{t x}\right)(t)\right\|^{2}+\int_{0}^{t}(1+\tau)^{3}\left\|y_{t t}\right\|^{2} d \tau \leq C \delta^{2} .
$$

Therefore, we obtain the following desired decay rates.

TheOREM 3.9. The solution y to (3.5) in Theorem 3.2 satisfies

$$
\sum_{k=0}^{1}\left[(1+t)^{k+1}\left\|\partial_{x}^{k} V(\cdot, t)\right\|^{2}+(1+t)^{k+2}\left\|\partial_{x}^{k} y_{t}(\cdot, t)\right\|^{2}\right] \leq C
$$

and

$$
\left\|y_{x}(\cdot, t)\right\|_{L^{\infty}} \leq C(1+t)^{-\frac{3}{4}},\left\|y_{t}(\cdot, t)\right\|_{L^{\infty}} \leq C(1+t)^{-\frac{5}{4}} .
$$

Proof. (3.32) comes directly from Lemmas 3.4-3.8. (3.33) follows from the interpolation inequality and (3.32), where

$$
\left\|y_{x}(\cdot, t)\right\|_{L^{\infty}} \leq C\|V(\cdot, t)\|_{L^{\infty}} \leq C(1+t)^{-\frac{3}{4}} .
$$

Theorem 3.1 then follows from Theorem 3.2 and Theorem 3.9.

4. Convergence to stationary solution. This section is devoted to proving Theorem 4 and Theorem 5 , where $\left(v_{-}, v_{+}\right)$and $\left(s_{-}, s_{+}\right)$are chosen so that $p\left(v_{-}, s_{-}\right)=$ $p\left(v_{+}, s_{+}\right)=\bar{p}=$ const.

Denote by $\left(v_{2}, u_{2}\right)(x, t)$ the solution of (1.4) obtained in Theorem 2.12. We solve (1.2)-(1.3) near $\left(v_{2}, u_{2}\right)(x, t)$ under $u_{-}=u_{+}=0$ and then (1.16) implies

$$
\int_{-\infty}^{+\infty}\left(v_{0}(x)-v_{2}(x, 0)\right) d x=0 .
$$

Similarly to section 3 , we set

$$
\tilde{y}=\int_{-\infty}^{x}\left(v(\xi, t)-v_{2}(\xi, t)\right) d \xi
$$


which satisfies

$$
\left\{\begin{array}{l}
\tilde{y}_{t t}+\left(p_{v}\left(v_{2}, s\right) \tilde{y}_{x}\right)_{x}+\tilde{y}_{t}=p\left(v_{2}, s\right)_{x t}-\left(F_{1}\left(v_{2}, \tilde{y}_{x}, s\right) \tilde{y}_{x}^{2}\right)_{x} \\
\tilde{y}(x, 0)=\tilde{y}_{0}(x)=\int_{-\infty}^{x}\left(v_{0}(\xi)-v_{2}(\xi, 0)\right) d \xi \\
\tilde{y}_{t}(x, 0)=\tilde{y}_{1}(x)=u_{0}(x)-u_{2}(x, 0)
\end{array}\right.
$$

where

$$
p\left(\tilde{y}_{x}+v_{2}, s\right)-p\left(v_{2}, s\right)=p_{v}\left(v_{2}, s\right) \tilde{y}_{x}+F_{1}\left(v_{2}, \tilde{y}_{x}, s\right) \tilde{y}_{x}^{2} .
$$

From the results in subsection 2.2 and the argument used in section 3, it is clear that the same argument of the section 3 can be used here to prove the following results.

THEOREM 4.1. There exists $\delta_{0}>0$ such that if $0<\delta<\delta_{0}$ and

$$
\left\|\tilde{y}_{0}\right\|_{3}^{2}+\left\|\tilde{y}_{1}\right\|_{2}^{2} \leq \delta
$$

then (4.2) has a unique smooth solution $\tilde{y}$ satisfying

$$
\sum_{k=0}^{1}\left[(1+t)^{k+1}\left\|\partial_{x}^{k} V_{1}(\cdot, t)\right\|^{2}+(1+t)^{k+2}\left\|\partial_{x}^{k} \tilde{y}_{t}(\cdot, t)\right\|^{2}\right] \leq C,
$$

with $V_{1}=p_{v}\left(v_{2}, s\right) \tilde{y}_{x}$, and

$$
\left\|\tilde{y}_{x}(\cdot, t)\right\|_{L^{\infty}} \leq C(1+t)^{-\frac{3}{4}},\left\|\tilde{y}_{t}(\cdot, t)\right\|_{L^{\infty}} \leq C(1+t)^{-\frac{5}{4}} .
$$

It is clear that Theorem 1.4 comes from Theorem 1.2 and Theorem 4.1.

We turn to proving Theorem 1.5 next. Since $\left(v_{1}(x), 0, s_{0}(x)\right)$ is the stationary solution of both (1.2) and (1.4), we can also solve (1.2)-(1.3) near $v_{1}$ instead of $v_{2}$, under the condition $u_{-}=u_{+}=0$ and (1.19), then

$$
\int_{-\infty}^{+\infty}\left(v_{0}(x)-v_{1}(x)\right) d x=0 .
$$

Denote

$$
z=\int_{-\infty}^{x}\left(v(\xi, t)-v_{1}(\xi)\right) d \xi
$$

then it follows that

$$
\left\{\begin{array}{l}
z_{t t}+\left(p_{v}\left(v_{1}, s\right) z_{x}\right)_{x}+z_{t}=-\left(F_{1}\left(v_{1}, z_{x}, s\right) z_{x}^{2}\right)_{x} \\
z(x, 0)=z_{0}(x)=\int_{-\infty}^{x}\left(v_{0}(\xi)-v_{1}(\xi)\right) d \xi \\
z_{t}(x, 0)=z_{1}(x)=u_{0}(x)
\end{array}\right.
$$

where

$$
p\left(z_{x}+v_{1}, s\right)-p\left(v_{1}, s\right)=p_{v}\left(v_{1}, s\right) z_{x}+F_{1}\left(v_{1}, z_{x}, s\right) z_{x}^{2} .
$$


We will prove the following theorem.

THEOREM 4.2. There exists $\delta_{0}>0$ such that if $0<\delta<\delta_{0}$ and

$$
\left\|z_{0}\right\|_{H^{3}}+\left\|z_{1}\right\|_{H^{2}} \leq \delta
$$

then (4.7) has a unique global smooth solution $z$ satisfying

$$
\sum_{k=0}^{1}\left[(1+t)^{k+1}\left\|\partial_{x}^{k} V_{2}(\cdot, t)\right\|^{2}+(1+t)^{k+2}\left\|\partial_{x}^{k} z_{t}(\cdot, t)\right\|^{2}\right] \leq C,
$$

where $V_{2}=p_{v}\left(v_{1}, s\right) z_{x}$ and

$$
\left\|z_{x}(\cdot, t)\right\|_{L^{\infty}} \leq C(1+t)^{-\frac{3}{4}},\left\|z_{t}(\cdot, t)\right\|_{L^{\infty}} \leq C(1+t)^{-\frac{5}{4}} .
$$

Hence (1.2)-(1.3) has a unique global smooth solution $(v, u, s)(x, t)$ such that

$$
\left\|\left(v-v_{1}\right)(\cdot, t)\right\|_{L^{\infty}} \leq C(1+t)^{-\frac{3}{4}},\|u(\cdot, t)\|_{L^{\infty}} \leq C(1+t)^{-\frac{5}{4}} .
$$

We note that Theorem 4.2 implies Theorem 1.5.

Using the same proof as in Theorem 3.2, noting $p\left(v_{1}, s\right)=$ const, we can deduce the following lemma.

Lemma 4.3. There exists $\delta_{0}>0$ such that if $0<\delta<\delta_{0}$ and

$$
\left\|z_{0}\right\|_{H^{3}}+\left\|z_{1}\right\|_{H^{2}} \leq \delta
$$

then (4.7) has a unique smooth solution $z$ satisfying

$$
\|z(t)\|_{H^{3}}^{2}+\left\|z_{t}(t)\right\|_{H^{2}}^{2}+\int_{0}^{t}\left\|\left(z_{x}, z_{t}\right)(\tau)\right\|_{H^{2}} d \tau \leq C \delta^{2} .
$$

The next result concerns the decay rates.

LEMma 4.4. The solution $z$ of (4.7), obtained in Lemma 4.3, satisfies

$$
\sum_{k=0}^{1}\left[(1+t)^{k+1}\left\|\partial_{x}^{k} V_{2}(\cdot, t)\right\|^{2}+(1+t)^{k+2}\left\|\partial_{x}^{k} z_{t}(\cdot, t)\right\|^{2}\right] \leq C,
$$

where $V_{2}=p_{v}\left(v_{1}, s\right) z_{x}$ and

$$
\left\|z_{x}(\cdot, t)\right\|_{L^{\infty}} \leq C(1+t)^{-\frac{3}{4}},\left\|z_{t}(\cdot, t)\right\|_{L^{\infty}} \leq C(1+t)^{-\frac{5}{4}} .
$$

Proof. We multiply $(4.7)_{1}$ by $(1+t) z_{t}$ and integrate it by parts. Then by using (4.8), we obtain, by a calculation similar to that one in the proof of Lemma 3.4, that

$$
(1+t)\left\|\left(z_{x}, z_{t}\right)(t)\right\|^{2}+\int_{0}^{t}(1+\tau)\left\|z_{t}(\tau)\right\|^{2} d \tau \leq C \delta^{2} .
$$

Now let us differentiate $(4.7)_{1}$ in $t$, then we have

$$
z_{t t t}+\left(p_{v}\left(v_{1}, s\right) y_{x}\right)_{x t}+y_{t t}=-\left(F_{1} z_{x}^{2}\right)_{x t} .
$$


Multiplying (4.12) by $(1+t) z_{t}$ and $(1+t) z_{t t}$, respectively, we deduce

$$
\begin{gathered}
{\left[(1+t)\left(z_{t} z_{t t}+\frac{1}{2} z_{t}^{2}\right)\right]_{t}-p_{v}\left(v_{1}, s\right)(1+t) z_{t x}^{2}-(1+t) z_{t t}^{2}} \\
=\frac{1}{2} z_{t}^{2}+z_{t} z_{t t}-\left(F_{1} z_{x}^{2}\right) t_{t}(1+t) z_{t x}+\{\cdots\}_{x}, \\
\quad\left[\frac{1}{2}(1+t)\left(z_{t t}^{2}-p_{v} z_{t x}^{2}\right)\right]_{t}+(1+t) z_{t t}^{2} \\
\left.=\frac{1}{2} z_{t t}^{2}-\frac{1}{2} p_{v} z_{t x}^{2}-(1+t) z_{t t x}\left(F_{1} z_{x}^{2}\right)_{t}\right)+\{\cdots\}_{x} .
\end{gathered}
$$

$$
\begin{aligned}
& (1+t)\left\|\left(z_{t t}, z_{t x}\right)\right\|^{2}+\int_{0}^{t}(1+\tau)\left\|\left(z_{t t}, z_{t x}\right)(\tau)\right\|^{2} d \tau \\
& \leq C+C\left|\int_{0}^{t} \int_{-\infty}^{+\infty}(1+\tau)\left(z_{t x}+z_{t t x}\right)\left(F_{1} z_{x}^{2}\right)_{t} d x d \tau\right| .
\end{aligned}
$$

We see that

$$
\begin{aligned}
& \left|\int_{0}^{t} \int_{-\infty}^{+\infty}(1+\tau) z_{t x}\left(F_{1} z_{x}^{2}\right)_{t} d x d \tau\right| \\
& \leq C \delta \int_{0}^{t}(1+\tau)\left\|z_{t x}(\tau)\right\|^{2} d \tau
\end{aligned}
$$

and

$$
\begin{aligned}
& \left|\int_{0}^{t} \int_{-\infty}^{+\infty}(1+\tau) z_{t t x}\left(F_{1} z_{x}^{2}\right)_{t} d x d \tau\right| \\
& \leq C\left|\int_{0}^{t} \int_{-\infty}^{+\infty}\left[O(1)\left|z_{x}\right|(1+\tau) z_{t x}^{2}\right]_{t} d x d \tau\right| \\
& \quad+C \delta \int_{0}^{t} \int_{-\infty}^{+\infty}(1+(1+\tau)) z_{t x}^{2} d x d \tau
\end{aligned}
$$

Due to the smallness of $\delta$, from (4.15)-(4.17) we have

$$
(1+t)\left\|\left(z_{t t}, z_{t x}\right)\right\|^{2}+\int_{0}^{t}(1+\tau)\left\|\left(z_{t t}, z_{t x}\right)(\tau)\right\|^{2} d \tau \leq C .
$$

Now let us multiply $(4.12)$ by $(1+t)^{2} z_{t}$ and $(1+t)^{2} z_{t t}$ and repeat the previous calculations, then

$$
(1+t)^{2}\left\|\left(z_{t}, z_{t t}, z_{t x}\right)\right\|^{2}+\int_{0}^{t}(1+\tau)^{2}\left\|\left(z_{t t}, z_{t x}\right)(\tau)\right\|^{2} d \tau \leq C .
$$

The same proof as used in Lemma 3.6 yields

$$
(1+t)^{2}\left\|\left(V_{2 t}, V_{2 x}\right)\right\|^{2}+\int_{0}^{t}(1+\tau)\left\|\left(V_{2 t}, V_{2 x}\right)(\tau)\right\|^{2} d \tau \leq C .
$$


By differentiating (4.12) in $x$, we get

$$
(1+t)^{2}\left\|\left(z_{t t x}, z_{t x x}\right)\right\|^{2}+\int_{0}^{t}(1+\tau)^{2}\left\|\left(z_{t t x}, z_{t x x}\right)(\tau)\right\|^{2} d \tau \leq C,
$$

and finally, by multiplying (4.12) by $(1+t)^{3} z_{t t}$, it follows that

$$
\begin{aligned}
& (1+t)^{3}\left\|\left(z_{t t}, z_{t x}\right)\right\|^{2}+\int_{0}^{t}(1+\tau)^{3}\left\|z_{t t}(\tau)\right\|^{2} d \tau \\
& \leq C+C\left|\int_{0}^{t} \int_{-\infty}^{+\infty}\left[O(1)\left|z_{x}\right|(1+\tau)^{3} z_{t x}^{2}\right]_{t} d x d \tau\right| \\
& \quad+C \delta \int_{0}^{t} \int_{-\infty}^{+\infty}(1+\tau)^{2} z_{t x}^{2} d x d \tau+C\left|\int_{0}^{t} \int_{-\infty}^{+\infty}(1+\tau)^{3} z_{t x}^{3} d x d \tau\right|,
\end{aligned}
$$

which implies

$$
\begin{aligned}
& (1+t)^{3}\left\|\left(z_{t t}, z_{t x}\right)\right\|^{2}+\int_{0}^{t}(1+\tau)^{3}\left\|z_{t t}(\tau)\right\|^{2} d \tau \\
& \leq C+C\left|\int_{0}^{t} \int_{-\infty}^{+\infty}(1+\tau)^{3} z_{t x}^{3} d x d \tau\right| .
\end{aligned}
$$

We have

$$
\begin{aligned}
& \left|\int_{0}^{t} \int_{-\infty}^{+\infty}(1+\tau)^{3} z_{t x}^{3} d x d \tau\right| \\
& \leq C \int_{0}^{t} \int_{-\infty}^{+\infty}(1+\tau)^{2} z_{t x}^{2}+(1+\tau)^{4} z_{t x}^{4} d x d \tau \\
& \leq C+C \int_{0}^{t}(1+\tau)^{2}\left(\left\|z_{t x}\right\|^{2}+\left\|z_{t x x}\right\|^{2}\right) d \tau \\
& \leq C
\end{aligned}
$$

Then from (4.22)-(4.23), it follows that

$$
(1+t)^{3}\left\|\left(z_{t t}, z_{t x}\right)\right\|^{2}+\int_{0}^{t}(1+\tau)^{3}\left\|z_{t t}(\tau)\right\|^{2} d \tau \leq C .
$$

Hence, (4.9) follows from the combination of (4.11), (4.19)-(4.21), and (4.24). The estimate (4.10) follows from (4.9).

By combining Lemmas 4.3 and 4.4, we complete the proof of the Theorem 4.2.

Acknowledgments. This work was completed when the second author was visiting the Dipartmento di Matematica Pura ed Applicata, Universita degli Studi di L'Aquila; he wishes to thank Professor Pierangelo Marcati and his group for their warm hospitality. Some ideas for this paper arose when Pan visited the Istituto per le Applicazioni del Calcolo of CNR and the Department of Mathematics of the University of Rome "La Sapienza"; he wishes to thank professor Roberto Natalini for his kind hospitality. 


\section{REFERENCES}

[1] C. M. Dafermos, A system of hyperbolic conservation laws with frictional damping, Z. Angew. Math. Phys., 46 (1995), pp. 294-307.

[2] C. J. Van Duyn And L. A. Peletier, Asymptotic behavior of solutions of a nonlinear diffusion equations, Arch. Rational Mech. Anal., 65 (1977), pp. 363-377.

[3] C. J. Van Duyn And L. A. Peletier, A class of similary solutions of the nonlinear diffusion equations, Nonlinear Anal., 1 (1977), pp. 223-233.

[4] L. HsiaO, Quasilinear Hyperbolic Systems and Dissipative Mechanisms, World Scientific, River Edge, NJ, 1997.

[5] L. HsiaO AND T. P. Liu, Convergence to nonlinear diffusion waves for solutions of a system of hyperbolic conservation laws with damping, Comm. Math. Phys., 143 (1992), pp. 599-605.

[6] L. HSiaO AND T. P. LiU, Nonlinear diffusive phenomena of nonlinear hyperbolic systems, Chinese Ann. Math. Ser B, 14 (1993), pp. 465-480.

[7] L. HsiaO And T. LuO, Nonlinear diffusive phenomena of solutions for the system of compressible adiabatic flow through porous media, J. Differential Equations, 125 (1996), pp. 329-365.

[8] L. Hsiao AND T. LuO, Nonlinear diffusive phenomena of entropy weak solutions for a system of quasilinear hyperbolic conservation laws with damping, Quart. Appl. Math., 56 (1998), pp. 173-198.

[9] L. HsiaO AND R. H. PAN, Initial boundary value problem for the system of compressible adiabatic flow through porous media, J. Differential Equations, 159 (1999), pp. 280-305.

[10] L. Hsiao AND D. SERre, Global existence of solutions for the system of compressible adiabatic flow through porous media, SIAM J. Math. Anal., 27 (1996), pp. 70-77.

[11] L. Hsiao AND D. SerRe, Large-time behavior of solutions for the system of comprssible adiabatic flow through porous media, Chinese Ann. Math. Ser. B, 16 (1995), pp. 1-14.

[12] L. HSIAO AND S. Q. TANG, Construction and qualitative behavior of solutions for a system of nonlinear hyperbolic conservation laws with damping, Quart. Appl. Math., 53 (1995), pp. $487-505$.

[13] L. HsiaO AND S. Q. TANG, Construction and qualitative behavior of the solution of the perturbated Riemann problem for the system of one-dimensional isentropic flow with damping, J. Differential Equations, 123 (1995), pp. 480-503.

[14] T. LuO AND T. YANG, Interaction of elementary waves for compressible Euler equations with frictional damping, J. Differential Equations, 161 (2000), pp. 42-86.

[15] P. Marcati and A. Milani, The one dimensional Darcy's law as the limit of a compressible Euler flow, J. Differential Equations, 84 (1990), pp. 129-147.

[16] P. Marcati AND M. MeI, Convergence to nonlinear diffusion waves for solutions of the initial boundary problem to the hyperbolic conservation laws with damping, Quart. Appl. Math., 53 (2000), pp. 763-784.

[17] P. Marcati, A. Milani, And P. Secchi, Singular convergence of weak solutions for a quasilinear nonhomogeneous hyperbolic system, Manuscripta Math., 60 (1988), pp. 49-69.

[18] P. Marcati and B. Rubino, Hyperbolic to parabolic relaxation theory for quasilinear first order systems, J. Differential Equations, 162 (2000), pp. 359-399.

[19] K. NishinaRa, Convergence rates to nonlinear diffusion waves for solutions of system of hyperbolic conservation laws with damping, J. Differential Equations, 131 (1996), pp. 171-188.

[20] K. Nishinara And T. YAng, Boundary effect on asymptotic behavior of solutions to the psystem with damping, J. Differential Equations, 161 (2000), pp. 191-218.

[21] D. Serre And L. XiaO, Asymptotic behavior of large weak entropy solutions of the damped p-system, J. Partial Differential Equations, 10 (1997), pp. 355-368.

[22] Y. S. ZHEng, Global smooth solutions to the adiabatic gas dynamics system with dissipation terms, Chinese J. Contemp. Math., 17 (1996), pp. 155-162. 